\title{
Toponimia relacionada con sabinas y enebros (Gén. Juniperus) en la España peninsular. Primera aproximación
}

\author{
Première approche à la toponymie basée sur les genévriers \\ (Gen. Juniperus) en Espagne péninsulaire
}

Luis VILLAR ${ }^{1}$, Mario SANZ²

1. Instituto Pirenaico de Ecología, IPE-CSIC, Apdo. 64, 22700 Jaca (Huesca, España)

E-mail : Ivillar@ipe.csic.es

2. Gerencia Territorial del Catastro, Ministerio de Hacienda y Administraciones Públicas, Segovia (España)

E-mail : mario.sanz@catastro.minhap.es

\section{Resumen}

El género Juniperus incluye 11 especies y subespecies en la Península Ibérica, 7 de hoja acicular y 4 de hoja escuamiforme. En España, sólo la especie principal -J. thurifera L.- ocupa más de 100.000 ha y encontramos sabinas o enebros desde 0 a $3000 \mathrm{~m}$ de altitud. Sus comunidades conforman hábitats priorizados para su conservación a escala europea y bastantes lugares de interés comunitario (LIC) llevan sus nombres. Por tratarse de plantas leñosas muy útiles han recibido decenas de nombres vernáculos, entre los cuales observamos casos de sinonimia y polisemia. También se han formado muchos topónimos juniperinos individuales o colectivos. De ellos hemos recopilado -con especial atención a Castilla y Aragón- 432 referidos a las tres especies de la sect. Sabina, J. phoenicea, J. sabina y J. thurifera. Junto al topónimo menor o mayor se anotan localidad, nombre científico de la especie que lo originó, provincia y comunidad autónoma. Desde un punto de vista etnobotánico, los topónimos estudiados indican referencia geográfica, utilidad -leñas y madera, alimento para herbívoros, abrigo, etc.- u otras características. Aunque en el siglo XXI se pierdan algunos de esos usos, los fitotopónimos persisten y ayudan a comprender la antigua explotación de esas formaciones forestales, su situación actual e incluso su evolución futura.

Palabras clave : fitotopónimos, Juniperus sect. Sabina, sinonimia-polisemia, etnoecología, España.

\section{Resumé}

Le genre Juniperus comprend onze taxons ibériques (sept à feuilles piquantes et quatre à feuilles en écaille). En Espagne, seul J. thurifera L. couvre beaucoup plus de 100000 ha mais les genévriers se distribuent depuis $0 \mathrm{~m}$ jusqu'à $3000 \mathrm{~m}$ d'altitude. Les junipéraies constituent des habitats prioritaires au niveau européen et de nombreux sites d'intérêt communautaire (SIC) portent leurs noms. Ces espèces ligneuses très utiles possèdent des dizaines de noms vernaculaires, parmi lesquels des synonymes et noms polysémiques. Surtout pour la Castille et l'Aragon, on retrouve ainsi beaucoup des toponymes "juniperains" individuels ou collectifs et nous en avons recensé 432 basés sur les espèces de la section Sabina, J. phoenicea, J. sabina et J. thurifera. À coté du nom de lieu nous avons noté les noms scientifiques des espèces concernées, la localité, province et région. Du point de vue ethnobotanique, les toponymes étudiés viennent indiquer une référence géographique, leur utilisation (bois, alimentation des herbivores, abri, etc.) ou d'autres caractéristiques. Même si, aujourd'hui, certains de ces usages sont en train de se perdre, les phytotoponymes persistent et nous rappellent l'intégration ancienne de I'homme dans son environnement. Leur étude peut nous aider à mieux comprendre l'exploitation ancienne de leurs formations forestières, leur situation actuelle et même leur évolution future.

Mots clés : phytotoponymes, Juniperus sect. Sabina, synonymie-polysémie, ethnoécologie. Espagne. 


\section{Introducción}

El género Juniperus (Cupressaceae) incluye en la Península Ibérica una docena de especies y subespecies mayormente conocidas como sabinas -con hoja adulta escuamiforme- y enebros, de hoja acicular (Villar 2013). Se trata de arbustos o árboles útiles al hombre para muy diversos fines o peligrosos en ciertos aspectos (Villar et al. 1987; Villar \& Ferrández 2000). En la Península Ibérica podemos encontrar sabinas desde el nivel del mar hasta los $2100 \mathrm{~m}$ y enebros hasta los $3000 \mathrm{~m}$ de altitud; la especie principal, $J$. thurifera L., endémica del Mediterráneo occidental, solo en Castilla y León ocupa más de 100000 ha (Yagüe et al. 2006). En España y otros países, estas leñosas ha sido aprovechadas desde tiempos prehistóricos (Costa et al. 1990) ; así, en unos lugares sus formaciones se fueron aclarando e incluso destruyendo, mientras que en otros se mantuvieron en el seno de un paisaje agro-silvo-pastoral. Ciertas especies definen comunidades vegetales características (Rivas-Martínez et al. 2011), ciertos ejemplares se consideran árboles singulares (Sanz \& Agón 1997) y algunas de sus masas son objeto de protección legal a escala regional. Aún más, en Europa conforman hábitats priorizados para su conservación y por tanto ayudan a delimitar lugares de interés comunitario (LIC), por ejemplo el llamado «Sabinares de la Sierra de Cabrejas», en Soria.

Castilla y León, junto con Aragón y territorios limítrofes, albergan todas las especies de Juniperus y aunque en ambas regiones se habla español, también siguen vivas otras lenguas o llegan influencias : gallego y portugués por el oeste; astur-leonés o bable por el noroeste ; aragonés y catalán por el este ; vascuence y gascón por el $\mathrm{N}$; árabe por el $\mathrm{S}$, etc.

En la Tabla 1 alistamos las cinco especies principales, de las cuales tres son de hoja adulta escuamiforme y dos de hoja acicular. Además de sus nombres científicos latinos hemos anotado las decenas de nombres vernáculos que han recibido en las diversas lenguas peninsulares (véanse Morales et al. 1996 ; Ruiz de la Torre 2006 ; Vidaller 2004 ; Villar et al. 1997-2001, etc.). Puede observarse que no existe correspondencia entre nombres cultos y vulgares sino frecuentes casos de sinonimia -varios nombres se dan a una sola especie- y de polisemia : una sola voz sirve para nombrar varias especies.
Esta dificultad llamó la atención de nuestro amigo el Prof. T. Gauquelin en el Coloquio de Soria, y su interés por el tema nos llevó a preparar esta comunicación.

Como era de esperar, sobre la base de ese conocimiento fitonímico, las poblaciones humanas han ido dejando a lo largo de los siglos numerosos topónimos individuales o colectivos (Villar 2005a y b ; Sanz \& González 2006, etc.). Aparte de su interés lingüístico, estas voces suelen llevar asociada información etnobotánica que nos interesa (Villar \& Ferrández 2000 ; Villar 2003).

En los últimos años venimos recogiendo en ese ámbito muchos topónimos mayores o menores relacionados con enebros y sabinas. Son fitotopónimos, sinfitotopónimos o hagiotopónimos con los que formamos las correspondientes bases de datos. En esta comunicación presentaremos una muestra de los topónimos juniperinos basados en taxones de la Sect. Sabina: Juniperus phoenicea, J. sabina y J. thurifera, es decir, aquellos que presentan hojas adultas escuamiformes.

Ante esta situación, en esta nota nuestros objetivos consisten en (1) averiguar la especie o especies en que se basan ; (2) interpretar el saber etnoecológico que reflejan (Villar 2010) en el seno de sus paisajes humanizados (González Bernáldez 1992; Barrios et al. 1992) -ya que no sólo denotan referencias geográficas sino también múltiples aprovechamientos en el espacio y en el tiempo: abrigo, obtención de leñas, madera de construcción, fabricación de muebles, utensilios y objetos, remedios medicinales o veterinarios, bebidas, repelente de insectos, alimento o complemento mineral para animales domésticos, ornamento, etc. - y (3) contribuir al esclarecimiento y conservación de este patrimonio cultural, ya que como dice Aguilella (2011) muestra una fragilidad o riesgo de pérdida todavía mayor que el de la información genética o ecológica. En efecto, tras la despoblación rural sufrida a lo largo de la segunda mitad del siglo XX, algunos de estos nombres perdieron vitalidad o cayeron en el olvido.

\section{Fuentes, material y métodos}

Nuestro conocimiento previo (Villar et al. 1997-2001; Sanz 2008) de los variados nombres vulgares de sabinas y enebros en el 
Tabla 1 - Especies del género Juniperus presentes en la Península Ibérica, con sus correspondientes nombres vulgares individuales, colectivos y principales usos etnobotánicos.

Table 1 - The Juniperus species living in the Iberian Peninsula, together with their correspondent popular names - individual and collective - and some ethnobotanical uses.

\begin{tabular}{|c|c|c|c|}
\hline Nombre cientítfico & $\begin{array}{l}\text { Nombres vulgares en la Península } \\
\text { lbérica y Baleares (individual) }\end{array}$ & $\begin{array}{l}\text { Nombres vulgares en la } \\
\text { Península Ibérica y Baleares } \\
\text { (colectivo) }\end{array}$ & Principales Usos \\
\hline \multirow[t]{11}{*}{ J. communis L. s.I. } & $\begin{array}{l}\text { Galaico/Portugués: cimbro, xenebreiro/zimbro, } \\
\text { zimbro-comum }\end{array}$ & $\begin{array}{l}\text { xenebral, xenebrerial, cimbral, } \\
\text { xinebreiral, jinebreiral, jiniebral/zimbral }\end{array}$ & $\begin{array}{l}\text { Artesanal, alimentación, } \\
\text { medicinal y veterinaria, } \\
\text { ornamental }\end{array}$ \\
\hline & $\begin{array}{l}\text { Español : enebro, enebro común, enebro real, } \\
\text { ginebro, grojo, jabino, sabino, sabina, sabina morisca }\end{array}$ & $\begin{array}{l}\text { enebral, enebredo, ginebral, nebral, } \\
\text { nebredo, sabinar }\end{array}$ & \\
\hline & $\begin{array}{l}\text { Castilla y León : árguma, carrasco, chaparro, enebra, } \\
\text { enebro, enebro de los pinchos, enhambri, enhembri, } \\
\text { jabina, jabino, sabino (algunas localidades de Sg), } \\
\text { churro (Valdivielso Bu), cimbro, xinibro, nebral, } \\
\text { nebro (Sayago Za), eneblio, enebrio (Montaña de } \\
\text { Luna Le), enebro (Sierra Demanda Bu ; Za) enebro } \\
\text { común (general), esquena, esqueno (algunas zonas } \\
\text { del centro y sur de Bu y P y de Va), grojo (valle Ebro } \\
\text { en Bu), inebro, jinebro, jineblo, jinieblo (Treviño Bu), } \\
\text { jinebrerira, jiniebra, xinibreira (Sanabria y Carballeda Za), } \\
\text { jiniebro (valle de Mena Bu), nebreira, nebrera } \\
\text { (La Cabrera y Valdería Le), xinebreira (La Cabrera Le), } \\
\text { xinebru (Montaña de Luna, Montaña de Riaño Le), } \\
\text { zainu (mamolar Bu) }\end{array}$ & $\begin{array}{l}\text { enebral, enebredo, nebral, nebredo, } \\
\text { sabinar }\end{array}$ & \\
\hline & $\begin{array}{l}\text { Otros: Aragonés: azotacristos, chinarro, chinastra, } \\
\text { chinebre, chinebre bord, chinebrero, chinebro, chinepro, } \\
\text { chinestra, chinibro, chinibra, chinipro, enebro, ginebro, } \\
\text { inebro, xinastra, xinebre, xinebro }\end{array}$ & $\begin{array}{l}\text { chinebral, chinipral, chiniprar, enebrada, } \\
\text { ginebral, ginebrar, inebrada, inebral }\end{array}$ & \\
\hline & Catalán : ginebre, ginebró & ginebreda & \\
\hline & $\begin{array}{l}\text { Vascuence : larraon, orre, orri, ipar, ipar ipurua, } \\
\text { ipar orrea, ipuru }\end{array}$ & orreadi, orreaga & \\
\hline & Otros: Gorbezu, gorbiñu, gurbañu (Asturias) & & \\
\hline & $\begin{array}{l}\text { Otros: Árabe : ârâr. Bereber : aífs, aîfs. Bereber: } \\
\text { tamerbut (ssp. hemisphaerica) }\end{array}$ & & \\
\hline & Otros: Aragonés: subsp. alpina : Chinebriza, chinepro & chinebral & \\
\hline & $\begin{array}{l}\text { Otros, subsp. alpina, Castilla y León : gorbizo, gorbizu, } \\
\text { (Montaña de Luna Le), gurbiezo (Montaña de Luna, } \\
\text { Montaña de Riaño); nebro, jimbro (Za) }\end{array}$ & & \\
\hline & $\begin{array}{l}\text { Otros, subsp. alpina : enebro de montaña (Andalucía) ; } \\
\text { garbizo (Asturias) }\end{array}$ & & \\
\hline \multirow[t]{6}{*}{ J. oxycedrus L. s.I. } & $\begin{array}{l}\text { Galaico/Portugués: -/Cedro-de-espanha, oxicedro, piorro, } \\
\text { zimbreira, zimbro-bravo, zimbro-molar }\end{array}$ & /jimbrial, junbrial, zimbral, zimbreiral & $\begin{array}{l}\text { Artesanal. Medicinal y } \\
\text { veterinario. Ritual. } \\
\text { Repelente de insectos. } \\
\text { Ornamental. Carbón }\end{array}$ \\
\hline & $\begin{array}{l}\text { Español: enebro, enebro de la miera, enebro español, } \\
\text { cada, cade, oxicedro }\end{array}$ & enebral & \\
\hline & $\begin{array}{l}\text { Castilla y León : boninas, la jimbre, jimbru, joimbre, } \\
\text { jumbrial, jumbrio, jimbro (Arribes del Duero en Sa y Za), } \\
\text { broja, grojo blanco (valle del Ebro en Bu), chaparro (algunas } \\
\text { localidades de Sg), chirrichín (algunas localidades } \\
\text { del norte So), enebro de la miera, nebro (algunas } \\
\text { localidades de Av), enhembre, jembre, jumbriu } \\
\text { (algunas localidades de Sa), espiteño Valle del Arlanza Bu), } \\
\text { esqueno, espiniego, esquino albarizo (algunas localidades } \\
\text { del oeste de Bu), inebru (Aliste Za), jabino mayor, sabino } \\
\text { (diversas áreas de Sg, Va y So), jimbrio (general), jiniebro } \\
\text { (algunas localidades del norte de So), nebru, niebro } \\
\text { (algunas localidades del oeste y norte de Le) }\end{array}$ & $\begin{array}{l}\text { enebral, enebredo, nebral, nebredo, } \\
\text { sabinar }\end{array}$ & \\
\hline & Castilla-La Mancha : enebro, nebro (Toledo) & & \\
\hline & $\begin{array}{l}\text { Otros: Aragonés: Azotacristos, chinebra, chinebre, } \\
\text { chinebrero, chinebro, chinebro de flor (macho), chinestra, } \\
\text { chinibro, chinipro, enebro, ginebro, inebro, jinebro, xinebre, } \\
\text { xinebro }\end{array}$ & enebril ? inebrada nebral? & \\
\hline & Catalán: càdec, càda & ginebreda? & \\
\hline
\end{tabular}


Vascuence : hego-ipurua, hego-orrea

Otros: Enebro albar (Andalucía)

Otros: Árabe : ârâr, araar. Tamazight o bereber :

aïfstaga, taqqa, tasnubar, tiqqi

Otros, subsp. badia : ajarje, azotacristo, enebro, nebro, pino, sestia (en Badajoz, Extremadura)

J. phoenicea L. s.l. Galaico/Portugués: -/Sabina-da-praia, sabina-das-areias, /sabinal, zimbreiral Artesanal. Medicinal y zimbro-das-areias, zimbreira da praia,

Español : sabina, sabina negra, sabina negral, sabina sabinal, sabinar

mora, sabina suave, sabina pudia

Castilla y León: alón, grojo romero (Las Merindades,

Montes Obarenes Bu), barda, enebro de barda (algunas

localidades del nordeste de So), enebro, enebro de risco

(valle del Ebro en Bu, algunas localidades del este de So),

minebra (Las Merindades Bu)

Otros: Aragonés: Pudia, sabina, sabina alta, sabina

de roca, sabina negra, sabina negral

Catalán: Savina; sivina (Baleares) savineda, savineta

Vascuence: Miter beltza, miter feniziarra, miterra,

sabina beltza, sabina feniziarra, sabina gorria

Otros : Sabina fenicia (Andalucía) sabinar

Otros : Árabe : ârâr, ârâr-ha-adom, ârâr-el-horr.

Bereber : aîfs

Otros, subsp. turbinata (Guss.) Nyman : Sabina marina

(español) ; savina marítima (catalán); sabina, sebina (Canarias)

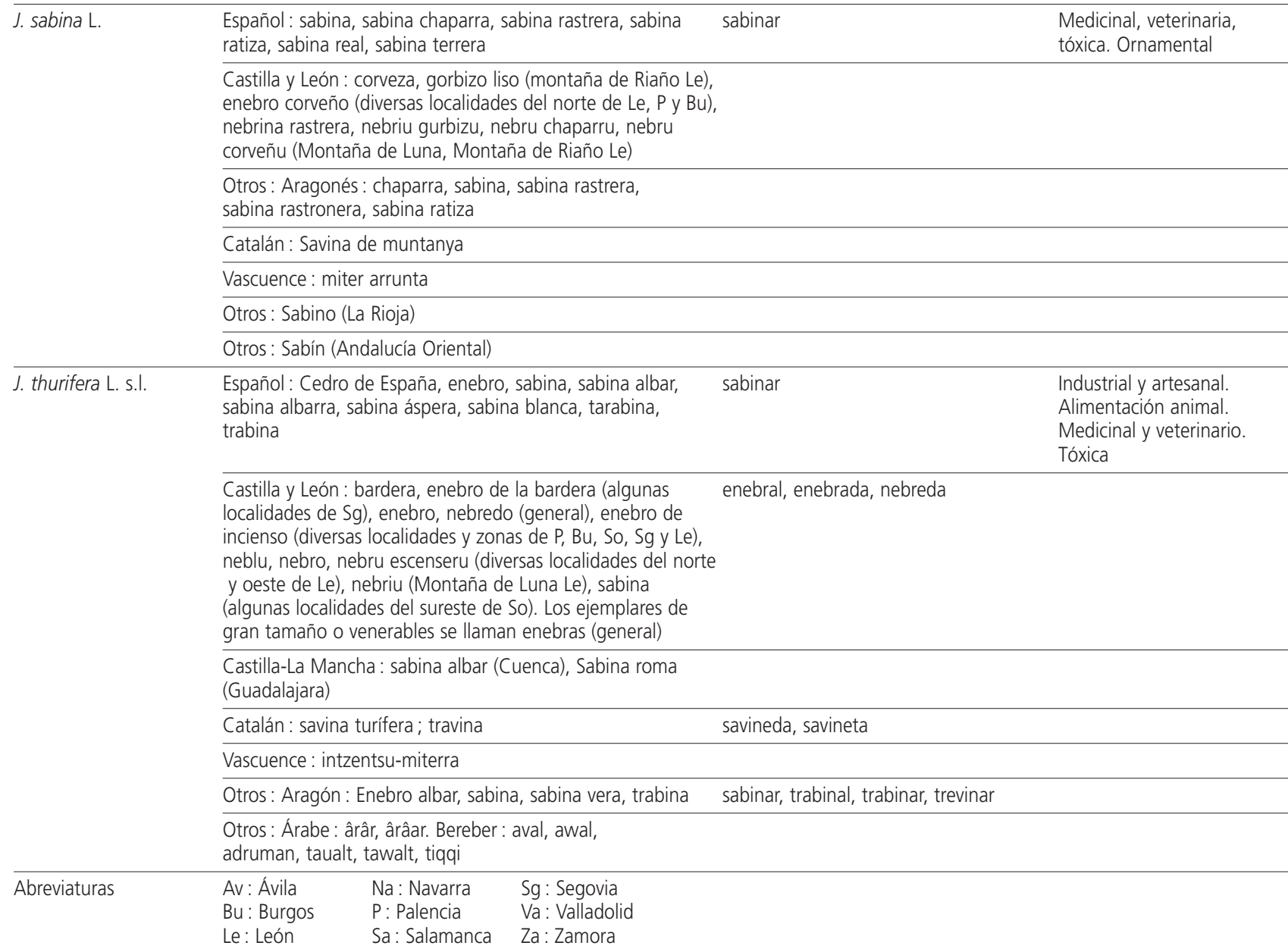


ámbito que nos ocupa se basaba en repertorios lexicográficos (Benito 2002), diccionarios (Vidaller 2004 ; Coromines 1989-1997) y en nuestra familiaridad con buena parte de las lenguas citadas. Pero además, varias fuentes nos han servido para extraer la información toponímica que buscábamos.

En primer lugar hemos revisado c. 120000 topónimos mayores -es decir, núcleos de población como El Sabinar, Valdenebro, etc., accidentes orográficos, hidrografía o vías de comunicación- en la base de datos georreferenciada de nombres geográficos NoMgEO, versión 29.09, del Instituto Geográfico Nacional, hoy en el Ministerio de Fomento. De entre ellos hemos anotado 8081 registros fitotoponímicos, de los cuales 159 corresponden a las especies de Juniperus. Asimismo incluimos algunos santuarios o ermitas relacionados, es decir, hagiotopónimos como la Ermita de la Virgen de Sabina o Nuestra Señora de los Enebrales (Morales \& Villar 2003).

En segundo lugar, respecto a los topónimos menores o microtopónimos, es decir, los nombres de parajes o pagos, empezamos por revisar nuestras bases de datos previas sobre fitotopónimos de Aragón (Villar 2005a y b ; Sanz \& Agón 1997) más Castilla y León (Sanz \& González 2008) o territorios limítrofes, consistentes en varios miles de registros. Luego acudimos a los nutridos datos toponímicos de la Dirección General del Catastro (Ministerio de Hacienda y Administraciones públicas), cedidos por las Gerencias Territoriales en forma de listados municipales. A ellos cabe añadir los catálogos provinciales de Montes Públicos, en el caso de Aragón actualizados para las provincias de Zaragoza, Huesca y Teruel (Pérez-Soba 2010; BOA 2011a, 2011b), más otras fuentes bibliográficas o documentales : mapas, atlas lingüísticos (Alvar et al. 1980, García Sánchez 2007, Gordaliza \& Canal, 1993) y monografías diversas. Así las cosas, el número de microtopónimos revisados sobrepasa los 700000 .

De unos y otros extrajimos los nombres relacionados con las tres especies citadas de Juniperus, ya sean fitotopónimos individuales -como Fuentenebro, Turó de la Savina Rodona (Aymerich \& Villar 2013) o Sabinazo-, sinfitotopónimos -bien sean plurales como Las Enebras o colectivos como Negredo, Enebral, Corvezal-, abundanciales como Sierra de Sabinós, etc.

Por este procedimiento confeccionamos una base de datos específica con más de 1100 registros, de los cuales aproximadamente el $55 \%$ corresponden a Castilla y León y el $45 \%$ a Aragón. Finalmente, de ella hemos seleccionado 432 basados en las especies de la Sect. Sabina. Junto al nombre del paraje o topónimo mayor, añadimos otros campos como provincia, municipio, entidad agregada y taxón o taxones de Juniperus implicados en su formación. El proceso puede parecer sencillo pero no está exento de dificultades y nos ha obligado a corregir los errores observados -de transcripción u otros-, a evitar repeticiones, a resolver dudas sobre la especie o especies a que se refiere el topónimo, a matizar o averiguar los conocimientos etnoecológicos subyacentes, a eliminar algún neotopónimo sin base etnobotánica tradicional, etc.

\section{Resultados y discusión}

En la Tabla 2 se presentan esos 432 fitotopónimos relacionados con las especies juniperinas de hoja escuamiforme, y su análisis nos permitirá algunos comentarios. Dentro de cada comunidad autónoma y provincia, los parajes o topónimos mayores se ordenan por orden alfabético y se añade luego el municipio o municipios correspondientes, para terminar con el taxón o taxones de Juniperus implicados en su formación.

Destaquemos en primer lugar cómo la voz enebro y sus variantes - procedente precisamente del latín Juniperus-, es la más polisémica del género, ya que puede referirse a sus cinco especies peninsulares, las dos de hoja acicular (J. communis s.l., J. oxycedrus s.l.) y las tres de hoja escuamiforme: J. thurifera s.l. mayormente, J. phoenicea s.l. alguna vez y J. sabina excepcionalmente.

Por otra parte, el nombre sabina -y derivados con la misma raíz : jabina, jabino, sabino, trabina $^{1}$, trevina- no se refiere solo a cualquiera de las tres especies de la Sect. Sabina (Juniperus thurifera s.l., J. phoenicea s.l. y $J$. sabina), como podríamos pensar, sino también -en algún caso- a las especies de hoja acicular (J. communis, J. oxycedrus) ; es decir, se muestra asimismo polisémico,

Por tanto, ante un topónimo determinado, en muchas ocasiones no sabemos con certeza a qué especie o especies debe asociarse. Las

1. Según el DraE significa « el fruto de la sabina », acepción que indica originaria de Andalucía. 
Tabla 2 - Relación de topónimos juniperinos mayores y menores recogidos en las provincias de Aragón (Huesca, Teruel y Zaragoza) más Castilla y León (Burgos, León, Palencia, Segovia, Soria y Valladolid) con indicación de la especie o especies en los que se basan.

Table 2 - List of plant place names based on the genus Juniperus - major and minor - collected in Aragón provinces (Huesca, Teruel, and Zaragoza) and Castilla y León (Burgos, León, Palencia, Segovia, Soria, and Valladolid provinces) with an indication of the correspondent species.

\begin{tabular}{|c|c|c|c|c|}
\hline $\begin{array}{l}\text { Comunidad } \\
\text { autónoma }\end{array}$ & Provincia & Topónimo & Municipios & Taxón \\
\hline Aragón & Huesca & Camino de la Sabina & Sariñena & Juniperus thurifera \\
\hline Aragón & Huesca & Camino de los Sabinales & Sariñena & $\begin{array}{l}\text { Juniperus phoeniceal } \\
\text { Juniperus thurifera }\end{array}$ \\
\hline Aragón & Huesca & Camino del Sabinal & Sena & $\begin{array}{l}\text { Juniperus phoeniceal } \\
\text { Juniperus thurifera }\end{array}$ \\
\hline Aragón & Huesca & La Estacada & Lalueza & $\begin{array}{l}\text { Juniperus phoenicea/ } \\
\text { Juniperus thurifera }\end{array}$ \\
\hline Aragón & Huesca & La Sabinosa & Castillonroy & Juniperus phoenicea \\
\hline Aragón & Huesca & La Sabinosa & Viacamp y Litera & Juniperus phoenicea \\
\hline Aragón & Huesca & Matabinas & Bernues & Juniperus phoenicea \\
\hline Aragón & Huesca & Puy Sabina & Alcubierre & Juniperus thurifera \\
\hline Aragón & Huesca & Sabina & Hoz y Costean, Barluenga, San Miguel de Cinca & Juniperus phoenicea \\
\hline Aragón & Huesca & Sabina & Lanaja, Torralba de Aragón & Juniperus thurifera \\
\hline Aragón & Huesca & Sabinal & Las Peñas de Riglos & Juniperus phoenicea \\
\hline Aragón & Huesca & Sabinal & Alcolea de Cinca, Huerto & $\begin{array}{l}\text { Juniperus phoeniceal } \\
\text { Juniperus thurifera }\end{array}$ \\
\hline Aragón & Huesca & Sabinal & Villanueva de Sigena & $\begin{array}{l}\text { Juniperus phoeniceal } \\
\text { Juniperus thurifera }\end{array}$ \\
\hline Aragón & Huesca & Sabinales & El Grado & Juniperus phoenicea \\
\hline Aragón & Huesca & Sabinales & Sariñena & $\begin{array}{l}\text { Juniperus phoeniceal } \\
\text { Juniperus thurifera }\end{array}$ \\
\hline Aragón & Huesca & Sabinar & Alberuela de Tubo & $\begin{array}{l}\text { Juniperus phoeniceal } \\
\text { Juniperus thurifera }\end{array}$ \\
\hline Aragón & Huesca & Sabinares & Nocito & Juniperus phoenicea \\
\hline Aragón & Huesca & Sabinera & Botaya & Juniperus phoenicea \\
\hline Aragón & Huesca & Sabinera & Triste & Juniperus phoenicea \\
\hline Aragón & Huesca & Sabinera & Veracruz & Juniperus phoenicea \\
\hline Aragón & Huesca & Sabineta & Albelda & Juniperus phoenicea \\
\hline Aragón & Huesca & Santa Sabina & El Grado & Juniperus phoenicea \\
\hline Aragón & Huesca & Saso Sabinar & Alcolea de Cinca & $\begin{array}{l}\text { Juniperus phoenicea/ } \\
\text { Juniperus thurifera }\end{array}$ \\
\hline Aragón & Huesca & Sobinol & Gerbe, Griébal & Juniperus phoenicea \\
\hline Aragón & Huesca & Valsabina & Blecua y Torres & Juniperus phoenicea \\
\hline Aragón & Huesca & Vereda del Sabinal & Sena & $\begin{array}{l}\text { Juniperus phoeniceal } \\
\text { Juniperus thurifera }\end{array}$ \\
\hline Aragón & Teruel & Barranco del Cerro Sabinas & Teruel & Juniperus thurifera \\
\hline Aragón & Teruel & Barranco del Sabinar & $\begin{array}{l}\text { Tronchon, Teruel, Riodeva, Nogueruelas, } \\
\text { Lagueruela }\end{array}$ & Juniperus thurifera \\
\hline Aragón & Teruel & Barranco del Travinarejo & Abejuela & Juniperus thurifera \\
\hline Aragón & Teruel & Barranco del Gayubar & Corbalan & Juniperus thurifera \\
\hline Aragón & Teruel & Cabezo la Sabina & Obon & Juniperus thurifera \\
\hline Aragón & Teruel & Camino al val del Sabinar & Los Olmos & Juniperus thurifera \\
\hline Aragón & Teruel & Camino de la Paridera del Sabinar & Blancas & Juniperus thurifera \\
\hline Aragón & Teruel & Camino de la Sabina & Villarquemado & $\begin{array}{l}\text { Juniperus thuriferal } \\
\text { Juniperus phoenicea }\end{array}$ \\
\hline Aragón & Teruel & Camino de los Coscollares & Cortes de Aragón & Juniperus phoenicea \\
\hline Aragón & Teruel & Camino de Sabinarejo & Santa Eulalia del Campo & Juniperus thurifera \\
\hline Aragón & Teruel & Camino de Sardera a Castellote & Castellote & Juniperus phoenicea \\
\hline Aragón & Teruel & Camino del Collado la Sabina & Veguillas de la Sierra & Juniperus thurifera \\
\hline Aragón & Teruel & Camino del Sabinar & $\begin{array}{l}\text { Riodeva, Nogueruelas, Calamocha, } \\
\text { Blancas, Albentosa }\end{array}$ & Juniperus thurifera \\
\hline Aragón & Teruel & Camino del Sabinar & Tronchon, Teruel & Juniperus thurifera \\
\hline Aragón & Teruel & Camino del Sabinarico & Fuentes de Rubielos & Juniperus thurifera \\
\hline Aragón & Teruel & Camino del Trevinarejo & Abejuela & Juniperus thurifera \\
\hline Aragón & Teruel & Cañada del Sabinar & Blancas & Juniperus thurifera \\
\hline Aragón & Teruel & Caseto Sabinar & La Puebla de Valverde & Juniperus thurifera \\
\hline Aragón & Teruel & Cerna & Calamocha & Juniperus thurifera \\
\hline Aragón & Teruel & Cerno & Escucha & Juniperus thurifera \\
\hline Aragón & Teruel & Cerrada del Sabinar & Torrijo del Campo & Juniperus thurifera \\
\hline Aragón & Teruel & Cerrada Sabinar & Alcala de la Selva & Juniperus thurifera \\
\hline Aragón & Teruel & Cerro Alto-Sabinar & Cuevas Labradas & Juniperus thurifera \\
\hline Aragón & Teruel & Clotes de Estacas & Albentosa & Juniperus phoenicea \\
\hline Aragón & Teruel & Collado la Sabina & Veguillas de la Sierra & Juniperus thurifera \\
\hline Aragón & Teruel & Corral de la Sabina & La Puebla de Valverde & Juniperus thurifera \\
\hline Aragón & Teruel & Corral del Sabinar & Nogueruelas & Juniperus thurifera \\
\hline Aragón & Teruel & Dehesa & Fortanete, Griegos, Guadalaviar & Juniperus thurifera \\
\hline Aragón & Teruel & Dehesa del Sabinar & Urrea de Gaen & Juniperus thurifera \\
\hline
\end{tabular}




\begin{tabular}{|c|c|c|c|c|}
\hline $\begin{array}{l}\text { Comunidad } \\
\text { autónoma }\end{array}$ & Provincia & Topónimo & Municipios & Taxón \\
\hline Aragón & Teruel & Dehesa Mayor & Griegos & Juniperus thurifera \\
\hline Aragón & Teruel & Fuente Sabina & Moscardón & Juniperus thurifera \\
\hline Aragón & Teruel & Gallubares & Alpeñés & Juniperus thurifera \\
\hline Aragón & Teruel & Gayubar & Manzanera & Juniperus thurifera \\
\hline Aragón & Teruel & Hoya de la Sabina & Albentosa & Juniperus thurifera \\
\hline Aragón & Teruel & Hoya de la Sabina & Valbona & Juniperus thurifera \\
\hline Aragón & Teruel & Hoyica Sabina & Vivel del Rio Martín & $\begin{array}{l}\text { Juniperus thuriferal } \\
\text { Juniperus phoenicea }\end{array}$ \\
\hline Aragón & Teruel & La Sabina & Samper de Calanda & Juniperus phoenicea \\
\hline Aragón & Teruel & Las Dehesas & Fortanete & Juniperus thurifera \\
\hline Aragón & Teruel & Llano Estacada & Alba del Campo & Juniperus phoenicea \\
\hline Aragón & Teruel & Loma del Trabinal & Tronchon & Juniperus thurifera \\
\hline Aragón & Teruel & Loma la Sabina Gorda & Manzanera & Juniperus thurifera \\
\hline Aragón & Teruel & Loma Sabina & Monteagudo del Castillo & Juniperus thurifera \\
\hline Aragón & Teruel & Los Gayubares & El Pobo & Juniperus thurifera \\
\hline Aragón & Teruel & Mup 218 Collado Sabina & Camarena de la Sierra & Juniperus thurifera \\
\hline Aragón & Teruel & Mup-21a « dehesa mayor» & Griegos & Juniperus thurifera \\
\hline Aragón & Teruel & Paso de la Sabina & Cella, Celadas & Juniperus thurifera \\
\hline Aragón & Teruel & Paso Ganados Sabinilla & La Puebla de Valverde & Juniperus thurifera \\
\hline Aragón & Teruel & Paso la Sabina Grande & Cortes de Aragón & Juniperus thurifera \\
\hline Aragón & Teruel & Pieza Sabina & Cella & Juniperus thurifera \\
\hline Aragón & Teruel & Prado Sabinotes & Royuela & Juniperus thurifera \\
\hline Aragón & Teruel & Rambla Sabina & Santa Eulalia del Campo & Juniperus thurifera \\
\hline Aragón & Teruel & Sabina & Calanda, Ejulve, Alcorisa & Juniperus phoenicea \\
\hline Aragón & Teruel & Sabina & Mas de las Matas & Juniperus phoenicea \\
\hline Aragón & Teruel & Sabina & $\begin{array}{l}\text { Veguillas de la Sierra, Fuenferrada, Montalban, } \\
\text { Monteaguno del Castillo, Moscardón, Torres } \\
\text { de Albarracín, Teruel, Noguera, Linares de } \\
\text { Mora, El Cuervo, Cosa, Cella, Castejón de } \\
\text { Tornos, Cascante del Río, Calamocha, } \\
\text { Bronchales, Arcos de las Salinas, Albentosa, } \\
\text { Abejuela }\end{array}$ & Juniperus thurifera \\
\hline Aragón & Teruel & Sabina & Villarquemado & $\begin{array}{l}\text { Juniperus thurifera/ } \\
\text { Juniperus phoenicea }\end{array}$ \\
\hline Aragón & Teruel & Sabina Bataneros & El Cuervo & Juniperus thurifera \\
\hline Aragón & Teruel & Sabina Blas & Veguillas de la Sierra & Juniperus thurifera \\
\hline Aragón & Teruel & Sabina Cacharro & Moscardón & Juniperus thurifera \\
\hline Aragón & Teruel & Sabina del Aire & Gea de Albarracin & Juniperus thurifera \\
\hline Aragón & Teruel & Sabina Gorda & Tormon, Cascante del Río & Juniperus thurifera \\
\hline Aragón & Teruel & Sabina Grande & Cortes de Aragón & Juniperus thurifera \\
\hline Aragón & Teruel & Sabina los Royos & Veguillas de la Sierra & Juniperus thurifera \\
\hline Aragón & Teruel & Sabina Morenos & Moscardón & Juniperus thurifera \\
\hline Aragón & Teruel & Sabina Podrida & Maicas & Juniperus thurifera \\
\hline Aragón & Teruel & Sabina Pudia & Alacon, Alloza & Juniperus phoenicea \\
\hline Aragón & Teruel & Sabina Redonda & Arcos de las Salinas & Juniperus thurifera \\
\hline Aragón & Teruel & Sabinago & Tormón & Juniperus thurifera \\
\hline Aragón & Teruel & Sabinal & La Ginebrosa, Torrijas, Oliete & Juniperus thurifera \\
\hline Aragón & Teruel & Sabinar & La Mata de los Olmos & Juniperus phoenicea \\
\hline Aragón & Teruel & Sabinar & $\begin{array}{l}\text { Hijar, Valbona, Urrea de Gaén, Alpeñes, } \\
\text { Utrillas, Torrijo del Campo, Teruel, Rubielos } \\
\text { de la Cérida, Royuela, Obón, Nogueruelas, } \\
\text { Mora de Rubielos, Los Olmos, La Puebla } \\
\text { de Valverde, EI Vallecillo, La Hoz de la Vieja, } \\
\text { Las Parras de Martín, Loscos, Sarrión, } \\
\text { Cuevas Labradas, Crivillén, Camarena } \\
\text { de la Sierra, Calamocha, Blancas, Arcos } \\
\text { de las Salinas, Alcalá de la Selva, Abejuela }\end{array}$ & Juniperus thurifera \\
\hline Aragón & Teruel & Sabinar cruz & Fuentes de Rubielos & Juniperus thurifera \\
\hline Aragón & Teruel & Sabinar del barranco lobo & Albarracin & Juniperus thurifera \\
\hline Aragón & Teruel & Sabinar del pozo & Mora de Rubielos & Juniperus thurifera \\
\hline Aragón & Teruel & Sabinar lobos & Abejuela & Juniperus thurifera \\
\hline Aragón & Teruel & Sabinarejo & $\begin{array}{l}\text { Veguillas de la Sierra, Toril y Masegoso, } \\
\text { Santa Eulalia del Campo, Royuela, } \\
\text { Arcos de las Salinas }\end{array}$ & Juniperus thurifera \\
\hline Aragón & Teruel & Sabinares & Alcañiz & Juniperus phoenicea \\
\hline Aragón & Teruel & Sabinares & $\begin{array}{l}\text { Veguillas de la Sierra, Josa, Royuela, } \\
\text { Arcos de las Salinas }\end{array}$ & Juniperus thurifera \\
\hline Aragón & Teruel & Sabinarico & Fuentes de Rubielos & Juniperus thurifera \\
\hline Aragón & Teruel & Sabinaricos & Teruel & Juniperus thurifera \\
\hline Aragón & Teruel & Sabinas negras & Loscos & Juniperus phoenicea \\
\hline Aragón & Teruel & Sabinasos & Valderrobres & $\begin{array}{l}\text { Juniperus thuriferal } \\
\text { Juniperus phoenicea }\end{array}$ \\
\hline Aragón & Teruel & Sabinaza & Veguillas de la Sierra & Juniperus thurifera \\
\hline Aragón & Teruel & Sabinazo & Veguillas de la Sierra & Juniperus thurifera \\
\hline
\end{tabular}




\begin{tabular}{|c|c|c|c|c|}
\hline $\begin{array}{l}\text { Comunidad } \\
\text { autónoma }\end{array}$ & Provincia & Topónimo & Municipios & Taxón \\
\hline Aragón & Teruel & Sabinejo & Toril y Masegoso & Juniperus thurifera \\
\hline Aragón & Teruel & Sabinica & Abejuela & Juniperus thurifera \\
\hline Aragón & Teruel & Sabinilla & Loscos & Juniperus phoenicea \\
\hline Aragón & Teruel & Sabinilla & $\begin{array}{l}\text { Veguillas de la Sierra, Torrijo del Campo, } \\
\text { Pozuel del Campo, La Puebla de Valverde, } \\
\text { Fonfría }\end{array}$ & Juniperus thurifera \\
\hline Aragón & Teruel & Sabinosa & Tramacastiel, Sarrión, Manzanera, Griegos & Juniperus thurifera \\
\hline Aragón & Teruel & Sabinoso & Tramacastiel & Juniperus thurifera \\
\hline Aragón & Teruel & Sabinotes & Royuela & Juniperus thurifera \\
\hline Aragón & Teruel & Santa Sabrina & Teruel & Juniperus thurifera \\
\hline Aragón & Teruel & Sardetas & Loscos & Juniperus phoenicea \\
\hline Aragón & Teruel & Senda de la Sabinosa & Sarrión & Juniperus thurifera \\
\hline Aragón & Teruel & Senda del Cabezo la Sabina & Obon & Juniperus thurifera \\
\hline Aragón & Teruel & Senda Sabinar & Teruel & Juniperus thurifera \\
\hline Aragón & Teruel & Senda Sabino & Cuevas Labradas & Juniperus thurifera \\
\hline Aragón & Teruel & Solano de la Sabina & Villarluengo & $\begin{array}{l}\text { Juniperus thurifera/ } \\
\text { Juniperus phoenicea }\end{array}$ \\
\hline Aragón & Teruel & Trevinarejo & Abejuela & Juniperus thurifera \\
\hline Aragón & Teruel & Val de Sabinar & Los Olmos & Juniperus thurifera \\
\hline Aragón & Teruel & Val Sabina & Ejulve & Juniperus phoenicea \\
\hline Aragón & Teruel & Vereda de Santa Sabinilla & La Puebla de Valverde & Juniperus thurifera \\
\hline Aragón & Zaragoza & Abejar Sabinos & Tarazona & Juniperus phoenicea \\
\hline Aragón & Zaragoza & Alto Sabina & Monreal de Ariza & Juniperus thurifera \\
\hline Aragón & Zaragoza & Arroyo del Coscojar & Sigüés & Juniperus phoenicea \\
\hline Aragón & Zaragoza & Barranco de Gayubar & Cimballa & Juniperus thurifera \\
\hline Aragón & Zaragoza & Barranco de la Sarda & Los Pintanos & Juniperus phoenicea \\
\hline Aragón & Zaragoza & Boquero Sabinoso & Perdiguera & Juniperus thurifera \\
\hline Aragón & Zaragoza & Camino de la Corona Sabinosa & Leciñena & Juniperus thurifera \\
\hline Aragón & Zaragoza & Camino de la Sabina & Abanto & Juniperus thurifera \\
\hline Aragón & Zaragoza & Camino de las Sardillas & Pina de Ebro & Juniperus phoenicea \\
\hline Aragón & Zaragoza & Camino de los Sabinales & Monegrillo & Juniperus thurifera \\
\hline Aragón & Zaragoza & Camino de Sabinales a Monegrillo & Monegrillo & Juniperus thurifera \\
\hline Aragón & Zaragoza & Camino de Val Sabinosa & Pina de Ebro & Juniperus thurifera \\
\hline Aragón & Zaragoza & Camino del Cerro de Val Sabinosa & Pina de Ebro & Juniperus thurifera \\
\hline Aragón & Zaragoza & Camino del Corral de la Sarda & Samper del Salz & Juniperus phoenicea \\
\hline Aragón & Zaragoza & Camino del Sabinar & Mediana de Aragón & Juniperus thurifera \\
\hline Aragón & Zaragoza & Camino del Sabinar & Castiliscar & Juniperus phoenicea \\
\hline Aragón & Zaragoza & Camino del Sabinoso & Ariza & Juniperus thurifera \\
\hline Aragón & Zaragoza & Camino Planar del Sabinar & Sástago & Juniperus thurifera \\
\hline Aragón & Zaragoza & Camino Sabina & Luna & Juniperus phoenicea \\
\hline Aragón & Zaragoza & Camino Varella de Sabina & Valmadrid & Juniperus thurifera \\
\hline Aragón & Zaragoza & Campo de la Sabina & Alborge & Juniperus thurifera \\
\hline Aragón & Zaragoza & Campo la Sabina & Valmadrid & Juniperus thurifera \\
\hline Aragón & Zaragoza & Cordel de las Sardillas & Pina de Ebro & Juniperus phoenicea \\
\hline Aragón & Zaragoza & El Sabinar (macrotopónimo) & Ejea de los Caballeros & Juniperus phoenicea \\
\hline Aragón & Zaragoza & El Sabinarejo & Calmarza & Juniperus thurifera \\
\hline Aragón & Zaragoza & El Sardon & Tosos & Juniperus phoenicea \\
\hline Aragón & Zaragoza & Enebrada & Sisamón & Juniperus thurifera \\
\hline Aragón & Zaragoza & Ermita ntra sra Virgen Sabina & Farlete & Juniperus thurifera \\
\hline Aragón & Zaragoza & Galluboso & Atea & Juniperus thurifera \\
\hline Aragón & Zaragoza & Gayubar & Olvés & Juniperus thurifera \\
\hline Aragón & Zaragoza & La Estacada & Alfajarin & Juniperus thurifera \\
\hline Aragón & Zaragoza & La Sabinilla & Berdejo & Juniperus thurifera \\
\hline Aragón & Zaragoza & La Sabinilla & Embid de Ariza & Juniperus thurifera \\
\hline Aragón & Zaragoza & La Sarda & San Mateo de Gállego & Juniperus phoenicea \\
\hline Aragón & Zaragoza & La Sarda Alta & San Mateo de Gállego & Juniperus phoenicea \\
\hline Aragón & Zaragoza & La Sarda Negra & Zaragoza & Juniperus phoenicea \\
\hline Aragón & Zaragoza & La Sardilla & Perdiguera & Juniperus phoenicea \\
\hline Aragón & Zaragoza & Las Sabinas & Lagata & Juniperus phoenicea \\
\hline Aragón & Zaragoza & Las Sardas & Farlete & Juniperus phoenicea \\
\hline Aragón & Zaragoza & Loma Sabina & Villamayor de Gállego & Juniperus thurifera \\
\hline Aragón & Zaragoza & Loma Sabina & Zaragoza & $\begin{array}{l}\text { Juniperus thuriferal } \\
\text { Juniperus phoenicea }\end{array}$ \\
\hline Aragón & Zaragoza & Los Sabinales & Monegrillo & Juniperus thurifera \\
\hline Aragón & Zaragoza & Morron del Coscojar & Used & Juniperus phoenicea \\
\hline Aragón & Zaragoza & Parada la Sabina & Zaragoza & Juniperus thurifera \\
\hline Aragón & Zaragoza & Paso Sabina & Torralba de los frailes & $\begin{array}{l}\text { Juniperus thurifera/ } \\
\text { Juniperus phoenicea }\end{array}$ \\
\hline Aragón & Zaragoza & Plana del Sabinar & Jaulín & Juniperus thurifera \\
\hline Aragón & Zaragoza & Puisabina & Zuera & Juniperus thurifera \\
\hline Aragón & Zaragoza & Sabina & Zaragoza & $\begin{array}{l}\text { Juniperus thurifera/ } \\
\text { Juniperus phoenicea }\end{array}$ \\
\hline Aragón & Zaragoza & Sabina & $\begin{array}{l}\text { Villamayor de Gállego, Villanueva de Huerva, } \\
\text { Torrehermosa, Bijuesca, La Almolda, Alhama } \\
\text { de Aragón, Abanto }\end{array}$ & Juniperus thurifera \\
\hline
\end{tabular}




\begin{tabular}{|c|c|c|c|c|}
\hline $\begin{array}{l}\text { Comunidad } \\
\text { autónoma }\end{array}$ & Provincia & Topónimo & Municipios & Taxón \\
\hline Aragón & Zaragoza & Sabina & Clarés de Ribota & Juniperus phoenicea \\
\hline Aragón & Zaragoza & Sabina & $\begin{array}{l}\text { Sestrica, Navardún, Morata de Jiloca, } \\
\text { Clarés de Ribota }\end{array}$ & Juniperus phoenicea \\
\hline Aragón & Zaragoza & Sabina Albar & Torrehermosa & Juniperus thurifera \\
\hline Aragón & Zaragoza & Sabina del Montero & Monegrillo & Juniperus thurifera \\
\hline Aragón & Zaragoza & Sabinal & Farlete & Juniperus thurifera \\
\hline Aragón & Zaragoza & Sabinal Hondo & Monegrillo & Juniperus thurifera \\
\hline Aragón & Zaragoza & Sabinar & Castiliscar & Juniperus phoenicea \\
\hline Aragón & Zaragoza & Sabinar & Plenas, Jaraba, Castiliscar & Juniperus phoenicea \\
\hline Aragón & Zaragoza & Sabinar & La Almolda, Abanto & Juniperus thurifera \\
\hline Aragón & Zaragoza & Sabinaro & Torralba de los Frailes & $\begin{array}{l}\text { Juniperus thurifera/ } \\
\text { Juniperus phoenicea }\end{array}$ \\
\hline Aragón & Zaragoza & Sabinilla & Torralba de los Frailes & $\begin{array}{l}\text { Juniperus thurifera/ } \\
\text { Juniperus phoenicea }\end{array}$ \\
\hline Aragón & Zaragoza & Sabinosa & Pina de Ebro & Juniperus thurifera \\
\hline Aragón & Zaragoza & Sabinosa & Lecera & $\begin{array}{l}\text { Juniperus thuriferal } \\
\text { Juniperus phoenicea }\end{array}$ \\
\hline Aragón & Zaragoza & Sabinoso & Moneva & Juniperus phoenicea \\
\hline Aragón & Zaragoza & Sabinoso & Ariza, Embid de Ariza & Juniperus thurifera \\
\hline Aragón & Zaragoza & Sarda & $\begin{array}{l}\text { Zuera, Villanueva de Gállego, Rueda de Jalón, } \\
\text { Pedrola, Nigüella, Murillo de Gállego, Mara, } \\
\text { Fuendejalón, Farlete }\end{array}$ & Juniperus phoenicea \\
\hline Aragón & Zaragoza & Sarda Baja & Zaragoza & Juniperus phoenicea \\
\hline Aragón & Zaragoza & Sarda Baladino & Sierra de Luna & Juniperus phoenicea \\
\hline Aragón & Zaragoza & Sarda Bona & Sierra de Luna & Juniperus phoenicea \\
\hline Aragón & Zaragoza & Sarda Cosme & $\begin{array}{l}\text { Perdiguera } \\
\text { Pera }\end{array}$ & Juniperus phoenicea \\
\hline Aragón & Zaragoza & Sarda Covatillas & Niguella & Juniperus phoenicea \\
\hline Aragón & Zaragoza & Sarda de Campo & Zaragoza & Juniperus phoenicea \\
\hline Aragón & Zaragoza & Sarda del Molino & Zaragoza & Juniperus phoenicea \\
\hline Aragón & Zaragoza & Sarda del Tejar & Zaragoza & Juniperus phoenicea \\
\hline Aragón & Zaragoza & Sarda Fuente & Niguella & Juniperus phoenicea \\
\hline Aragón & Zaragoza & Sarda Hornaz & Tauste & Juniperus phoenicea \\
\hline Aragón & Zaragoza & Sarda las Vacas & Sierra de Luna, Nigüella & Juniperus phoenicea \\
\hline Aragón & Zaragoza & Sarda Madero & Sierra de Luna & Juniperus phoenicea \\
\hline Aragón & Zaragoza & Sarda Marcial & Perdiguera & Juniperus phoenicea \\
\hline Aragón & Zaragoza & Sarda Soltera & Zaragoza & Juniperus phoenicea \\
\hline Aragón & Zaragoza & Sardera & Fuendejalón & Juniperus phoenicea \\
\hline Aragón & Zaragoza & Sardeta & Sástago, Pedrola & Juniperus phoenicea \\
\hline Aragón & Zaragoza & Senda de la Sabina & Abanto & Juniperus thurifera \\
\hline Aragón & Zaragoza & Senda de las Sabinas & Lagata & Juniperus phoenicea \\
\hline Aragón & Zaragoza & Senda Sabinas Común & Samper del Salz & $\begin{array}{l}\text { Juniperus thuriferal } \\
\text { Juniperus phoenicea }\end{array}$ \\
\hline Aragón & Zaragoza & Umbria del Gayubal & Ariza & Juniperus thurifera \\
\hline Aragón & Zaragoza & Vago Sabina & Belmonte de Gracián & Juniperus thurifera \\
\hline Aragón & Zaragoza & Val Sabinosa & Pina de Ebro & Juniperus thurifera \\
\hline Aragón & Zaragoza & Valdesabina & Biota & Juniperus phoenicea \\
\hline Aragón & Zaragoza & Varella Sabina & Valmadrid & Juniperus thurifera \\
\hline Aragón & Zaragoza & Vereda de la Sabina & El Frago & Juniperus phoenicea \\
\hline Aragón & Zaragoza & Vereda de la Sarda & Plenas & Juniperus phoenicea \\
\hline Castilla y León & Burgos & Arroyo de la Enebra & Tubilla del Lago & Juniperus thurifera \\
\hline Castilla y León & Burgos & Arroyo de Nebreda & Nebreda, Solarana & Juniperus thurifera \\
\hline Castilla y León & Burgos & Arroyo Negredales & Merindad de Río Ubierna & Juniperus thurifera \\
\hline Castilla y León & Burgos & Arroyo Negredo & Quintanapalla, Merindad de Río Ubierna & Juniperus thurifera \\
\hline Castilla y León & Burgos & Camino de Aceitenebro & Contreras, Barbadillo del Mercado & Juniperus thurifera \\
\hline Castilla y León & Burgos & Camino de Fuentenebro & Moradillo de Roa & Juniperus thurifera \\
\hline Castilla y León & Burgos & Camino de Fuentenebro & Torregalindo & Juniperus thurifera \\
\hline Castilla y León & Burgos & Camino de la Enebra & Lerma, Revillarruz & Juniperus thurifera \\
\hline Castilla y León & Burgos & Camino de la Fuente de la Enebra & Gumiel de Izán & Juniperus thurifera \\
\hline Castilla y León & Burgos & Camino de los Tres Enebros & Torresandino & Juniperus thurifera \\
\hline Castilla y León & Burgos & Camino de Nebreda & $\begin{array}{l}\text { Ciruelos de Cervera, Lerma, Cebrecos, } \\
\text { Solarana, Pineda-Trasmonte, Tejada, Valdeande }\end{array}$ & Juniperus thurifera \\
\hline Castilla y León & Burgos & Camino de Negredales & Merindad de Río Ubierna & Juniperus thurifera \\
\hline Castilla y León & Burgos & Camino del Enebral & Arauzo de Torre, Lerma & Juniperus thurifera \\
\hline Castilla y León & Burgos & Camino del Enebron & Mambrillas de Lara & Juniperus thurifera \\
\hline Castilla y León & Burgos & Camino del Enebrón & Caleruega & Juniperus thurifera \\
\hline Castilla y León & Burgos & Carrefuentenebro & Torregalindo & Juniperus thurifera \\
\hline Castilla y León & Burgos & Carrelosenebros & Solarana & Juniperus thurifera \\
\hline Castilla y León & Burgos & Carrenebrada & Solarana & Juniperus thurifera \\
\hline Castilla y León & Burgos & Carrenebreda & Quintanilla del Agua-Tordueles & Juniperus thurifera \\
\hline Castilla y León & Burgos & Cauce de Enebro & Roa & Juniperus thurifera \\
\hline Castilla y León & Burgos & Colada del Enebrillo al Corral & Bahabón de Esgueva & Juniperus thurifera \\
\hline Castilla y León & Burgos & Colector de Nebreda & Lerma & Juniperus thurifera \\
\hline Castilla y León & Burgos & El Enebro Alto & Fuentelcésped & Juniperus thurifera \\
\hline Castilla y León & Burgos & Enebra & $\begin{array}{l}\text { Castrillo de la Vega, Covarrubias, Lerma, } \\
\text { Oquillas, Sotillo de la Ribera, Tubilla del Lago, } \\
\text { Villalba de Duero }\end{array}$ & Juniperus thurifera \\
\hline
\end{tabular}




\begin{tabular}{|c|c|c|c|c|}
\hline $\begin{array}{l}\text { Comunidad } \\
\text { autónoma }\end{array}$ & Provincia & Topónimo & Municipios & Taxón \\
\hline Castilla y León & Burgos & Enebrada & $\begin{array}{l}\text { Caleruega, Carcedo de Bureba, Fuentelcésped, } \\
\text { Hontoria de Valdearados, Mambrillas de la Jara, } \\
\text { Nebreda, Pineda-Trasmonte, La Vid, Solarana }\end{array}$ & Juniperus thurifera \\
\hline Castilla y León & Burgos & Enebradillas & Santo Domingo de Silos & Juniperus thurifera \\
\hline Castilla y León & Burgos & Enebrados & Avellanosa de Muño & Juniperus thurifera \\
\hline Castilla y León & Burgos & Enebrajo & Santa María del Campo & Juniperus thurifera \\
\hline Castilla y León & Burgos & Enebral & $\begin{array}{l}\text { Arauzo de Torre, Caleruega, Carazo, Fresneña, } \\
\text { Galbarros, Hortigüela, Huerta del Rey, Los Altos } \\
\text { Nebreda, Puentedura, Quintana del Pidio, } \\
\text { Quintanilla del Agua-Tordueles, Valdorros, } \\
\text { Villamayor de los Montes }\end{array}$ & Juniperus thurifera \\
\hline Castilla y León & Burgos & Enebral & Merindad de Valdivielso, Poza de la Sal & Juniperus phoenicea \\
\hline Castilla y León & Burgos & Enebral & Valle de Mena & Juniperus phoenicea \\
\hline Castilla y León & Burgos & Enebral de Alla & Huerta de Rey & Juniperus thurifera \\
\hline Castilla y León & Burgos & Enebrales & $\begin{array}{l}\text { Fresneda de la Sierra, Santa María del Campo, } \\
\text { Santibáñez del Val, Valle de Valdelaguna, Zazua }\end{array}$ & Juniperus thurifera \\
\hline Castilla y León & Burgos & Enebralillo & Espinosa de Cervera & Juniperus thurifera \\
\hline Castilla y León & Burgos & Enebras & Cabañes de Esgueva & Juniperus thurifera \\
\hline Castilla y León & Burgos & Enebrazo & Arauzo de Miel & Juniperus thurifera \\
\hline Castilla y León & Burgos & Enebrillo & Bahabón de Esgueva, Haza & Juniperus thurifera \\
\hline Castilla y León & Burgos & Enebrillo & Moncalvillo, Pedrosa de Duero & Juniperus thurifera \\
\hline Castilla y León & Burgos & Enebrillo & Valle de Mena & Juniperus phoenicea \\
\hline Castilla y León & Burgos & Enebrillos & Mambrillas de Lara & Juniperus thurifera \\
\hline Castilla y León & Burgos & Enebro & $\begin{array}{l}\text { Fuentelcesped, Lerma, Moradillo de Roa, } \\
\text { Quintanilla del Agua-Tordueles, San Juan } \\
\text { del Monte, Villangomez, Villanueva de Gumiel }\end{array}$ & Juniperus thurifera \\
\hline Castilla y León & Burgos & Enebro la Pedrosa & Santo Domingo de Silos & Juniperus thurifera \\
\hline Castilla y León & Burgos & Enebro Portillo & Moncalvillo & Juniperus thurifera \\
\hline Castilla y León & Burgos & Enebro Pudio & Moncalvillo & Juniperus thurifera \\
\hline Castilla y León & Burgos & Enebro Redondo & Cebrecos & Juniperus thurifera \\
\hline Castilla y León & Burgos & Enebro Ruyales & Moncalvillo & Juniperus thurifera \\
\hline Castilla y León & Burgos & Enebro Sancho & Quintanilla del Coco & Juniperus thurifera \\
\hline Castilla y León & Burgos & Enebro Santo & Fontioso & Juniperus thurifera \\
\hline Castilla y León & Burgos & Enebron & Caleruega, Moncalvillo, Peñaranda de Duero & Juniperus thurifera \\
\hline Castilla y León & Burgos & Enebrones & Mambrillas de Lara & Juniperus thurifera \\
\hline Castilla y León & Burgos & Enebropobres & Cilleruelo de Arriba & Juniperus thurifera \\
\hline Castilla y León & Burgos & Enebros & $\begin{array}{l}\text { Pineda-Trasmonte, Valle de Valdelaguna, } \\
\text { Villalbilla de Gumiel, Villamayor de los Montes, } \\
\text { Villaverde del Monte }\end{array}$ & Juniperus thurifera \\
\hline Castilla y León & Burgos & Enebros Pobres & Nebreda & Juniperus thurifera \\
\hline Castilla y León & Burgos & Fuente de la Enebra & Gumiel de Izán & Juniperus thurifera \\
\hline Castilla y León & Burgos & Fuente del Enebro & Santo Domingo de Silos & Juniperus thurifera \\
\hline Castilla y León & Burgos & Fuente Enebrillo & La Vid & Juniperus thurifera \\
\hline Castilla y León & Burgos & Fuente Nebrillo & Valle de Tobalina & Juniperus phoenicea \\
\hline Castilla y León & Burgos & Fuentenebro (macrotopónimo) & Fuentenebro & Juniperus thurifera \\
\hline Castilla y León & Burgos & La Javinada & Arandilla, Peñaranda de Duero & $\begin{array}{l}\text { Juniperus communis/ } \\
\text { Juniperus oxycedrus }\end{array}$ \\
\hline Castilla y León & Burgos & La Negreda & Cabia & Juniperus thurifera \\
\hline Castilla y León & Burgos & La Sabina del Val & Huerta de Rey & Juniperus thurifera \\
\hline Castilla y León & Burgos & Los Nibriales & Monasterio de la Sierra & Juniperus thurifera \\
\hline Castilla y León & Burgos & Monte Negredo & Cubillo del Campo & Juniperus thurifera \\
\hline Castilla y León & Burgos & Montenegro & Cardeñadijo & Juniperus thurifera \\
\hline Castilla y León & Burgos & Nebraleja & Nebreda & Juniperus thurifera \\
\hline Castilla y León & Burgos & Nebreda & $\begin{array}{l}\text { Covarrubias, Lerma, Peñaranda de Duero, } \\
\text { Valdeande }\end{array}$ & Juniperus thurifera \\
\hline Castilla y León & Burgos & Nebreda (macrotopónimo) & Nebreda & Juniperus thurifera \\
\hline Castilla y León & Burgos & Nebrelos & Castildelgado & Juniperus thurifera \\
\hline Castilla y León & Burgos & Negradela & Huerta de Arriba & Juniperus thurifera \\
\hline Castilla y León & Burgos & Negrales & Villasandino & Juniperus thurifera \\
\hline Castilla y León & Burgos & Negredal & Los Balbases & Juniperus thurifera \\
\hline Castilla y León & Burgos & Negredales & Merindad de Río Ubierna & Juniperus thurifera \\
\hline Castilla y León & Burgos & Negredales & Sargentes de la Lora & Juniperus thurifera \\
\hline Castilla y León & Burgos & Negredales de Villafria & Sargentes de la Lora & Juniperus thurifera \\
\hline Castilla y León & Burgos & Negredas & Atapuerca, Villadur de Herreros & Juniperus thurifera \\
\hline Castilla y León & Burgos & Negredas & Quintanapalla & Juniperus thurifera \\
\hline Castilla y León & Burgos & Negredas de Zaluen & Atapuerca & Juniperus thurifera \\
\hline Castilla y León & Burgos & Negredos & $\begin{array}{l}\text { Arcos, Barrios de Colina, Cardeñajimeno, } \\
\text { Los Ausines, Ibeas de Juarros, Quintana-Vivar, } \\
\text { Burgos, Merindad de Río Ubierna, Valle } \\
\text { de Santibáñez, Villadiego, Villasur de Herreros }\end{array}$ & Juniperus thurifera \\
\hline Castilla y León & Burgos & Negredos de Castejon & Cardeñuela de Rio Pico & Juniperus thurifera \\
\hline Castilla y León & Burgos & Negredos de Valdemuñoz & Cardeñuela de Rio Pico & Juniperus thurifera \\
\hline Castilla y León & Burgos & Peña Negredas & Atapuerca & Juniperus thurifera \\
\hline Castilla y León & Burgos & Quiroñes Monte Nebreda & Nebreda & Juniperus thurifera \\
\hline Castilla y León & Burgos & Raya Monte Negredo & Madrigal del Monte & Juniperus thurifera \\
\hline
\end{tabular}




\begin{tabular}{|c|c|c|c|c|}
\hline $\begin{array}{l}\text { Comunidad } \\
\text { autónoma }\end{array}$ & Provincia & Topónimo & Municipios & Taxón \\
\hline Castilla y León & Burgos & Sabina & Pineda-Trasmonte, Urbel del Castillo & Juniperus thurifera \\
\hline Castilla y León & Burgos & Sabinillas & Quintanilla San-Garcia & Juniperus thurifera \\
\hline Castilla y León & Burgos & Senda de la Enebra & Terradillos de Esgueva & Juniperus thurifera \\
\hline Castilla y León & Burgos & Senda del Enebro & Huerta de Rey & Juniperus thurifera \\
\hline Castilla y León & Burgos & Tres Enebros & Torresandino & Juniperus thurifera \\
\hline Castilla y León & Burgos & Valdenebreda & Arauzo de Salce & Juniperus thurifera \\
\hline Castilla y León & Burgos & Valdenebro & Valle de Mena & Juniperus phoenicea \\
\hline Castilla y León & Burgos & Valdenegro & Coruña del Conde & Juniperus thurifera \\
\hline Castilla y León & Burgos & Valle de la Enebra & Lerma & Juniperus thurifera \\
\hline Castilla y León & León & Corvezal & Acebedo & Juniperus sabina \\
\hline Castilla y León & León & Corvezas & Villablino & Juniperus sabina \\
\hline Castilla y León & León & Nebredos & Crémenes & Juniperus thurifera \\
\hline Castilla y León & León & Nebrera & Toreno & Juniperus sabina \\
\hline Castilla y León & León & Nebrinas & Valderas & Juniperus sabina \\
\hline Castilla y León & León & Savinales & Villares de Órbigo & Juniperus thurifera \\
\hline Castilla y León & Palencia & Camino de Valdenebro & Belmonte de Campos, Reinoso de Cerrato & Juniperus thurifera \\
\hline Castilla y León & Palencia & Camino del Enebral & Villaconancio & Juniperus thurifera \\
\hline Castilla y León & Palencia & Camino del Enebral a Cobos & Tabanera de Cerrato & Juniperus thurifera \\
\hline Castilla y León & Palencia & Camino del Enebrillo & Cevico de la Torre, Villahán & Juniperus thurifera \\
\hline Castilla y León & Palencia & Camino del Negredo & Palenzuela & Juniperus thurifera \\
\hline Castilla y León & Palencia & Corvezones & Velilla del Rio Carrion & Juniperus sabina \\
\hline Castilla y León & Palencia & Enebral & $\begin{array}{l}\text { Antigüedad, Cevico Navero, Tabanera } \\
\text { de Cerrato, Villaconancio }\end{array}$ & Juniperus thurifera \\
\hline Castilla y León & Palencia & Enebrillo & Castrillo de Onielo, Soto de Cerrato & Juniperus thurifera \\
\hline Castilla y León & Palencia & Enebrillo & $\begin{array}{l}\text { Cevico Navero, Palenzuela, Quintana } \\
\text { del Puente, Tabanera de Cerrato, Villahán }\end{array}$ & Juniperus thurifera \\
\hline Castilla y León & Palencia & Enebro & Amusco, Hontoria de Cerrato & Juniperus thurifera \\
\hline Castilla y León & Palencia & La Sabina & Meneses de Campos & Juniperus thurifera \\
\hline Castilla y León & Palencia & Los Negrales & Poblacion de Cerrato & Juniperus thurifera \\
\hline Castilla y León & Palencia & Los Negredos & Lantadilla & Juniperus thurifera \\
\hline Castilla y León & Palencia & Nebrillo & Cevico Navero & Juniperus thurifera \\
\hline Castilla y León & Palencia & Negredo & Grijota, Palenzuela, Villahán & Juniperus thurifera \\
\hline Castilla y León & Palencia & Valdenebro & Reinoso de Cerrato & Juniperus thurifera \\
\hline Castilla y León & Segovia & Enebrada & $\begin{array}{l}\text { Castroserracín, Laguna de Contreras, } \\
\text { Cedillo de la Torre }\end{array}$ & Juniperus thurifera \\
\hline Castilla y León & Segovia & Enebradas & Navares de Ayuso, Prádena & Juniperus thurifera \\
\hline Castilla y León & Segovia & Enebrajos & Castrojimeno & Juniperus thurifera \\
\hline Castilla y León & Segovia & Enebral & $\begin{array}{l}\text { Arcones, Ayllón, Casla, Castro de Fuentidueña, } \\
\text { Fuenterrebollo, Otero de Herreros, Prádena, } \\
\text { Santo Tomé del Puerto, Torreadrada }\end{array}$ & Juniperus thurifera \\
\hline Castilla y León & Segovia & Enebralejo & $\begin{array}{l}\text { Castrojimeno, Santo Tomé del Puerto, } \\
\text { Ventosilla y Tejadilla }\end{array}$ & Juniperus thurifera \\
\hline Castilla y León & Segovia & Enebralejos & $\begin{array}{l}\text { Pradena, san Miguel de Bernuy, Villaverde } \\
\text { Montejo y Villalbilla }\end{array}$ & Juniperus thurifera \\
\hline Castilla y León & Segovia & Enebras & Fresno de Cantespino & Juniperus thurifera \\
\hline Castilla y León & Segovia & Enebrillo & $\begin{array}{l}\text { Arahuetes, Calabazas, Corral de Ayllón, } \\
\text { Languilla, Sepúlveda }\end{array}$ & Juniperus thurifera \\
\hline Castilla y León & Segovia & Enebrillos & Olombrada, Sepúlveda & Juniperus thurifera \\
\hline Castilla y León & Segovia & Enebrillos & Duruelo & Juniperus thurifera \\
\hline Castilla y León & Segovia & Enebro & $\begin{array}{l}\text { Armuña, Fuente el Olmo de Fuentidueña, } \\
\text { Melque de Cercos, Olombrada, Otero } \\
\text { de Herreros, Sacramenia, Santa María } \\
\text { la Real de Nieva }\end{array}$ & Juniperus thurifera \\
\hline Castilla y León & Segovia & Enebro Alto & Fuentesoto & Juniperus thurifera \\
\hline Castilla y León & Segovia & Enebro Gordo & Orejana & Juniperus thurifera \\
\hline Castilla y León & Segovia & Enebro Grande & Navalilla & Juniperus thurifera \\
\hline Castilla y León & Segovia & Enebro Mojon & Santo Tomé del Puerto & Juniperus thurifera \\
\hline Castilla y León & Segovia & Enebron & $\begin{array}{l}\text { Castroserracín, Montejo de la Vega } \\
\text { de la Serrezuela, Navares de las Cuevas }\end{array}$ & Juniperus thurifera \\
\hline Castilla y León & Segovia & Enebros & Fresno de Cantespino, Navalilla, Torredarada & Juniperus thurifera \\
\hline Castilla y León & Segovia & Enebros Altos & Castroserracín & Juniperus thurifera \\
\hline Castilla y León & Segovia & Enebros Grandes & Santo Tomé del Puerto & Juniperus thurifera \\
\hline Castilla y León & Segovia & Enebros Grandes & Valleruela de Pedraza & Juniperus thurifera \\
\hline Castilla y León & Segovia & Enebros Nuevos & Navalilla & Juniperus thurifera \\
\hline Castilla y León & Segovia & Enebros Viejos & Navalilla & Juniperus thurifera \\
\hline Castilla y León & Soria & Arroyo Enebrillo & Valderrodilla & Juniperus thurifera \\
\hline Castilla y León & Soria & Arroyo Fuente Enebro & Valderrodilla & Juniperus thurifera \\
\hline Castilla y León & Soria & Camino de Cabeza de Enebro & Retortillo de Soria & Juniperus thurifera \\
\hline Castilla y León & Soria & Camino de la Enebrada & Herrera de Soria & Juniperus thurifera \\
\hline Castilla y León & Soria & Camino de los Enebrillos & Fuente Cambrón & Juniperus thurifera \\
\hline Castilla y León & Soria & Camino del Enebral & Burgo de Osma-Ciudad de Osma & Juniperus thurifera \\
\hline Castilla y León & Soria & Camino del Enebro & Retortillo de Soria, San Esteban de Gormaz & Juniperus thurifera \\
\hline Castilla y León & Soria & Camino del Sabinar & Magaña, Santa María de Huerta & Juniperus thurifera \\
\hline Castilla y León & Soria & Camino Fuente Enebrillo & Montejo de Tiermes & Juniperus thurifera \\
\hline Castilla y León & Soria & Cañada Sabina Campo & Arcos de Jalón & Juniperus thurifera \\
\hline
\end{tabular}




\begin{tabular}{|c|c|c|c|c|}
\hline $\begin{array}{l}\text { Comunidad } \\
\text { autónoma }\end{array}$ & Provincia & Topónimo & Municipios & Taxón \\
\hline Castilla y León & Soria & Cerrada la Sabinilla & Medinaceli & Juniperus thurifera \\
\hline Castilla y León & Soria & Cerratas del Enebral & Montejo de Tiermes & Juniperus thurifera \\
\hline Castilla y León & Soria & Chozo del Sabinar & Magaña & Juniperus thurifera \\
\hline Castilla y León & Soria & Corral Negrillo & Villasayas & Juniperus thurifera \\
\hline Castilla y León & Soria & Corral Sabina & Coscurita & Juniperus thurifera \\
\hline Castilla y León & Soria & Corrales del Enebral & Montejo de Tiermes & Juniperus thurifera \\
\hline Castilla y León & Soria & Enebrada & $\begin{array}{l}\text { Burgo de Osma-Ciudad de Osma, Espejón, } \\
\text { Langa de Duero, Montejo de Tiermes }\end{array}$ & Juniperus thurifera \\
\hline Castilla y León & Soria & Enebradas & Espejón, Talveila & Juniperus thurifera \\
\hline Castilla y León & Soria & Enebradilla & Bayubas de Abajo & Juniperus thurifera \\
\hline Castilla y León & Soria & Enebral & $\begin{array}{l}\text { Burgo de Osma-Ciudad de Osma, Herrera } \\
\text { de Soria, Montejo de Tiermes, Quiñonería, } \\
\text { Retortillo de Soria, Valdeprado }\end{array}$ & Juniperus thurifera \\
\hline Castilla y León & Soria & Enebralejo & Talveila, Tardelcuende & Juniperus thurifera \\
\hline Castilla y León & Soria & Enebrales & $\begin{array}{l}\text { Burgo de Osma-Ciudad de Osma, Fresno } \\
\text { de Caracena, Espeja de San Marcelino, } \\
\text { Quiñonería }\end{array}$ & Juniperus thurifera \\
\hline Castilla y León & Soria & Enebrazo & Calatañazor & Juniperus thurifera \\
\hline Castilla y León & Soria & Enebrillas & Langa de Duero & Juniperus thurifera \\
\hline Castilla y León & Soria & Enebrillo & $\begin{array}{l}\text { Burgo de Osma-Ciudad de Osma, Carrascosa } \\
\text { de Abajo, Espeja de San Marcelino, Montejo } \\
\text { de Tiermes, Quintana Redonda, Recuerda, } \\
\text { Retortillo de Soria, San Esteban de Gormaz }\end{array}$ & Juniperus thurifera \\
\hline Castilla y León & Soria & Enebrillos & Fuente Cambrón, Langa de Duero & Juniperus thurifera \\
\hline Castilla y León & Soria & Enebro & $\begin{array}{l}\text { Berlanga de Duero, Burgo de Osma-Ciudad } \\
\text { de Osma, Golmayo, San Esteban de Gormaz }\end{array}$ & Juniperus thurifera \\
\hline Castilla y León & Soria & Enebron & Montejo de Tiermes & Juniperus thurifera \\
\hline Castilla y León & Soria & Enebros & Valdemaluque & Juniperus thurifera \\
\hline Castilla y León & Soria & Enebrote & Burgo de Osma-Ciudad de Osma & Juniperus thurifera \\
\hline Castilla y León & Soria & Eras Sabinilla & Arcos de Jalón & Juniperus thurifera \\
\hline Castilla y León & Soria & Fuente Enebrillo & Montejo de Tiermes & Juniperus thurifera \\
\hline Castilla y León & Soria & Fuente Enebro & Valderrodilla & Juniperus thurifera \\
\hline Castilla y León & Soria & Hondo Jabinos & San Esteban de Gormaz & $\begin{array}{l}\text { Juniperus communis/ } \\
\text { Juniperus oxycedrus }\end{array}$ \\
\hline Castilla y León & Soria & Hoya Enebrillo & San Esteban de Gormaz & Juniperus thurifera \\
\hline Castilla y León & Soria & Hoya Sabina & Arcos de Jalón, Medinaceli & Juniperus thurifera \\
\hline Castilla y León & Soria & Huerta Sabinilla & Arcos de Jalón & Juniperus thurifera \\
\hline Castilla y León & Soria & Llano Sabina & Monteagudo de las Vicarías & $\begin{array}{l}\text { Juniperus thurifera/ } \\
\text { Juniperus phoenicea }\end{array}$ \\
\hline Castilla y León & Soria & Llano Sabinar & Arcos de Jalón & Juniperus thurifera \\
\hline Castilla y León & Soria & Llano Sabino & Deza & $\begin{array}{l}\text { Juniperus thurifera/ } \\
\text { Juniperus phoenicea }\end{array}$ \\
\hline Castilla y León & Soria & Loma Negredo & Cabrejas del Campo & Juniperus thurifera \\
\hline Castilla y León & Soria & Monte Enebral & Cabrejas del Pinar, Tardelcuende & Juniperus thurifera \\
\hline Castilla y León & Soria & Negredillo & Viana de Duero & Juniperus thurifera \\
\hline Castilla y León & Soria & Negredo & Cabrejas del Campo, Garray & Juniperus thurifera \\
\hline Castilla y León & Soria & Negredos & $\begin{array}{l}\text { Garray, Hinojosa del Campo, Pinilla } \\
\text { del Campo, Viana de Duero }\end{array}$ & Juniperus thurifera \\
\hline Castilla y León & Soria & Negrera & Renieblas & Juniperus thurifera \\
\hline Castilla y León & Soria & Negrero & Soria & Juniperus thurifera \\
\hline Castilla y León & Soria & Prado del Enebro & Rioseco de Soria & Juniperus thurifera \\
\hline Castilla y León & Soria & Puntal la Sabinilla & Medinaceli & Juniperus thurifera \\
\hline Castilla y León & Soria & Sabina & Almenar de Soria, Medinaceli & Juniperus thurifera \\
\hline Castilla y León & Soria & Sabina & Cihuela, Monteagudo de las Vicarías & $\begin{array}{l}\text { Juniperus thuriferal } \\
\text { Juniperus phoenicea }\end{array}$ \\
\hline Castilla y León & Soria & Sabina Mocha & Arcos de Jalón & Juniperus thurifera \\
\hline Castilla y León & Soria & Sabinada & Valdegeña & $\begin{array}{l}\text { Juniperus thuriferal } \\
\text { Juniperus phoenicea }\end{array}$ \\
\hline Castilla y León & Soria & Sabinal & Cigudosa & $\begin{array}{l}\text { Juniperus thuriferal } \\
\text { Juniperus phoenicea }\end{array}$ \\
\hline Castilla y León & Soria & Sabinal & Ágreda & $\begin{array}{l}\text { Juniperus sabinal } \\
\text { Juniperus thurifera }\end{array}$ \\
\hline Castilla y León & Soria & Sabinal & Arcos de Jalón, Castilruiz & Juniperus thurifera \\
\hline Castilla y León & Soria & Sabinal & Cerbón & $\begin{array}{l}\text { Juniperus thurifera/ } \\
\text { Juniperus phoenicea }\end{array}$ \\
\hline Castilla y León & Soria & Sabinal Alto & Castilruiz & Juniperus thurifera \\
\hline Castilla y León & Soria & Sabinar & $\begin{array}{l}\text { Arcos de Jalón, Magaña, Matalebreras, } \\
\text { Santa Cruz de Yanguas, Santa María } \\
\text { de Huerta, Valdelagua del Cerro }\end{array}$ & Juniperus thurifera \\
\hline Castilla y León & Soria & Sabinares & Ciria & $\begin{array}{l}\text { Juniperus thuriferal } \\
\text { Juniperus phoenicea }\end{array}$ \\
\hline Castilla y León & Soria & Sabinazo & Arcos de Jalón & Juniperus thurifera \\
\hline Castilla y León & Soria & Sabinilla & Medinaceli & Juniperus thurifera \\
\hline Castilla y León & Soria & Sabinillo & Magaña & Juniperus thurifera \\
\hline
\end{tabular}




\begin{tabular}{|c|c|c|c|c|}
\hline $\begin{array}{l}\text { Comunidad } \\
\text { autónoma }\end{array}$ & Provincia & Topónimo & Municipios & Taxón \\
\hline Castilla y León & Soria & Sabino & Deza & $\begin{array}{l}\text { Juniperus thuriferal } \\
\text { Juniperus phoenicea }\end{array}$ \\
\hline Castilla y León & Soria & Senda de los Negredos & Hinojosa del Campo & Juniperus thurifera \\
\hline Castilla y León & Soria & Valdenebro (macrotopónimo) & Valdenebro & Juniperus thurifera \\
\hline Castilla y León & Soria & Valdenebroso & Langa de Duero & Juniperus thurifera \\
\hline Castilla y León & Soria & Valnebrillo & Burgo de Osma-Ciudad de Osma & Juniperus thurifera \\
\hline Castilla y León & Valladolid & Camino de la Enebrada & Viloria del Henar & Juniperus thurifera \\
\hline Castilla y León & Valladolid & Camino de Valdenebro & $\begin{array}{l}\text { Castromonte, Medina de Rioseco, Peñaflor } \\
\text { de Hornija, Villaverde de Campos, Villalba } \\
\text { de Los Alcores }\end{array}$ & Juniperus thurifera \\
\hline Castilla y León & Valladolid & Camino del Enebro & Villanueva de los Infantes & Juniperus thurifera \\
\hline Castilla y León & Valladolid & El Enebrillo & Langayo & Juniperus thurifera \\
\hline Castilla y León & Valladolid & Enebrada & Pesquera de Duero, Viloria del Henar & Juniperus thurifera \\
\hline Castilla y León & Valladolid & Enebral & Valbuena de Duero & Juniperus thurifera \\
\hline Castilla y León & Valladolid & Enebro & Bahabon, Íscar, Villanueva de los Infantes & Juniperus thurifera \\
\hline Castilla y León & Valladolid & Sendero Negredo & Traspinedo & Juniperus thurifera \\
\hline Castilla y León & Valladolid & Sote enebral & La Parrilla & Juniperus thurifera \\
\hline Castilla y León & Valladolid & $\begin{array}{l}\text { Valdenebro de los Valles } \\
\text { (macrotopónimo) }\end{array}$ & Valdenebro de los Valles & Juniperus thurifera \\
\hline
\end{tabular}

posibilidades de que se refiera a varias de ellas a la vez son muy altas sobre todo en el Centro-Norte, Sistema Ibérico y Valle del Ebro, ya que pueden coincidir dos especies e incluso sus híbridos en la misma localidad. Son casos llamativos de polisemia si tenemos en cuenta que el mismo topónimo o similar puede aludir a formaciones arbóreas, arbustivas, matorrales e incluso a masas mixtas de unas y otros.

Para mayor abundamiento, esa sinonimia múltiple -cerca de 20 nombres vernáculos para la sabina albar, véase Tabla 1 - nos lleva a casi todos los casos posibles de confusión entre los diversos topónimos generados. Por eso nos hemos visto obligados a anotar con frecuencia los dos taxones a que puede referirse el fitotopónimo. Cierto es que cuando se sitúan los fitotopónimos en el mapa y se cotejan con las áreas de distribución de las especies de hoja escamiforme (Ruiz de la Torre 2006) pueden resolverse algunas dudas. Por ejemplo, en ciertas localidades, un nombre designa inequívocamente a una de las especies ; así, el Racó de la Savina de Gandía (litoral valenciano) sólo puede señalar a J. phoenicea, mientras que en montes calizos del Sistema Ibérico, esa especie predomina a baja altitud, en tierras más altas $J$. thurifera va sola o en compañía de J. sabina. Aún más, esta última es común en lomas y áreas cimeras, desde el Moncayo a Gúdar y Javalambre, precisamente donde ya no sube $J$. thurifera. Otras veces el topónimo es compuesto; entonces, al unir al nombre un calificativo certero y excluyente se torna diáfano: Sabina Pudia, Sabina Mora, Sabinas Negras -los tres ejemplos de J. phoenicea-, Sabina Albar (J. thurifera), etc.

En el caso concreto de $J$. thurifera, árbol que se extiende desde León y Castilla hasta La Rioja y Navarra, Aragón, Cataluña y Valencia, más Murcia, Almería y Granada, al anotar sus nombres en tan vasto dominio comprobamos que por el centro y oeste de Castilla y León se le llama mayormente enebro -de donde enebredas, enebradas, nebredas, negredos, etc. - y en menor medida jabino. Opuestamente, en la parte oriental de Soria y Guadalajara, más los antiguos territorios de la Corona de Aragón, Murcia, Andalucía, Cuenca y Ciudad Real se le llama sabina o trabina, dando topónimos como sabinar, sabinal, trabinal, sabineta, etc. Teniendo en cuenta que en los Alpes de Alta Provenza su nombre antiguo es $\operatorname{serbin}^{2}$, como hipótesis se plantea que la influencia de la lengua occitana haya llegado al aragonés, se haya difundido por los antiguos territorios de la Corona de Aragón y haya podido alcanzar -quizá por los múltiples Caminos de Santiago ${ }^{3}$ - tierras castellanas orientales. Esta inversión semántica, ya conocida en los medios forestales, ha sido tratada con algún detalle recientemente (Coronado 2006) y nosotros la ilustramos en el mapa adjunto (Figura 1).

2. Nos preguntamos si podría indicar árbol que da frutos como los del serbal (server en catalán) pero con un matiz despectivo, pues no son comestibles.

3. Los lingüistas han encontrado modismos del aragonés en los primeros documentos de San Millán de la Cogolla, cuna del español, los cuales se pueden explicar por el hecho de que este cenobio dependía del Monasterio aragonés de San Juan de la Peña, ambos situados en la ruta jacobea. 


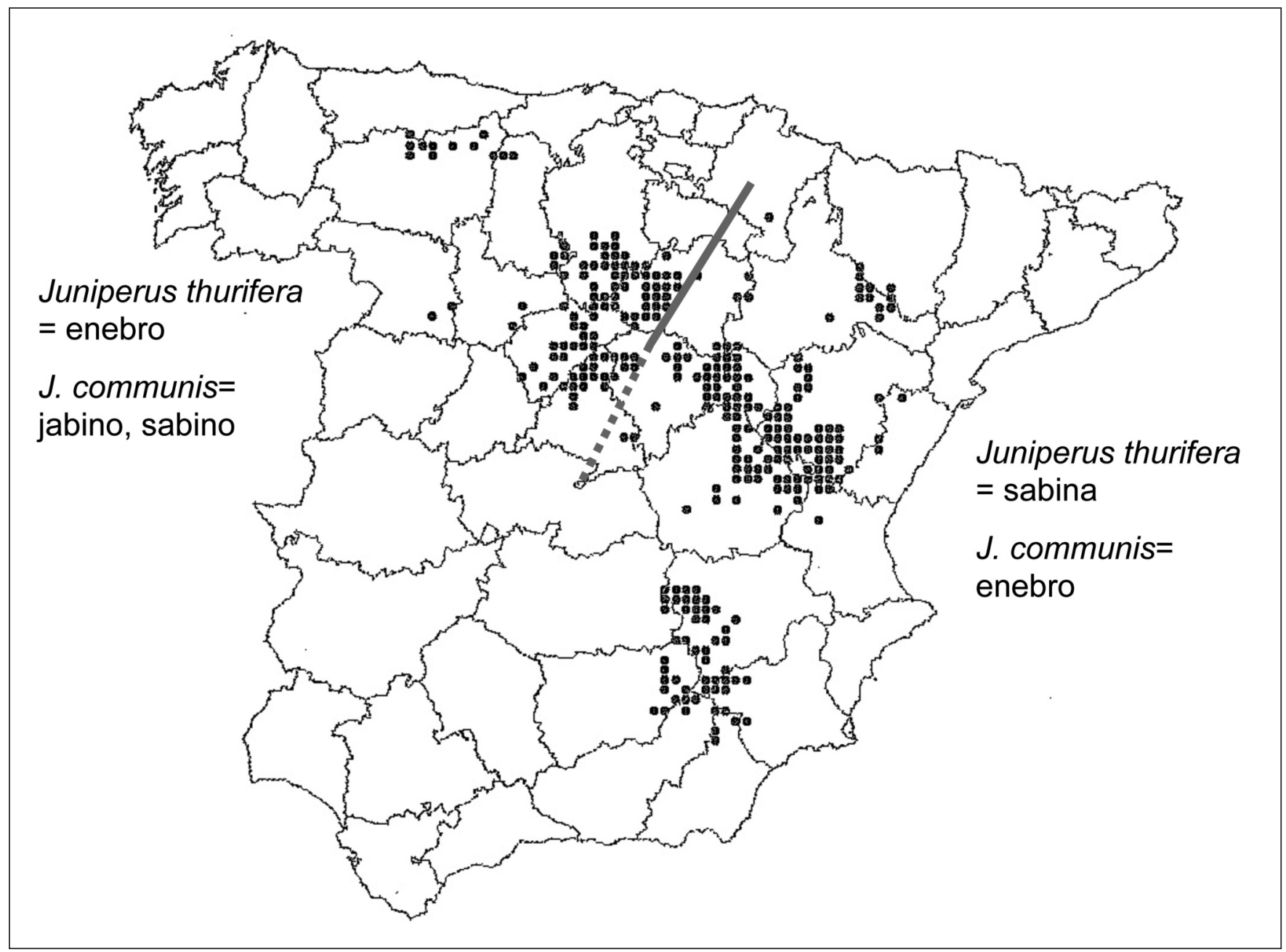

Figura 1 - Inversión semántica de las voces enebro y sabina para nombrar a Juniperus thurifera/J. communis en Castilla y León al W por oposición a Aragón, Cataluña, Valencia y otras regiones del E y S de España.

Figure 1 - Semantic inversion of the names enebro and Sabina to Juniperus thurifera/J. communis in Western Castilia when compared with Aragon, Catalonia, Valencia ando other regions of E and S Spain.

Los topónimos pueden atribuir el género masculino o femenino a una misma especie (jabino-sabina ; enebro-enebra) y ello puede tener connotaciones de edad o tamaño; en efecto, ejemplares viejos o grandes se suelen asociar al femenino. También se dan aumentativos (Enebrón) o diminutivos (Sabinillas, El Enebrillo). Los fitotopónimos relativos a individuos o a pequeños grupos suelen ser bastante transparentes. Unas veces sirven de referencia espacial -Puysabina, en Alcubierre (Huesca); Collado Sabina, en Camarena, (Teruel); Mas de la Sabina, en Valencia-, otras llevan determinativos de propiedad -Sabina de Blas, en Veguillas (Teruel)-, calificativos de forma-Sabina Redonda, en Arcos de las Salinas (Teruel)-, etc.

Sin embargo, los nombres colectivos o sinfitotopónimos ya son algo más difíciles, al llevar sufijos y terminaciones diversas según la lengua de que se trate. Así, por ejemplo, el sufijo latino -etum puede dar sinfitotopónimos en -edo (Negredo) -eda (Valdenebreda) por toda Castilla, etc., algún -ito en Aragón (Yenefrito, derivado de Genebrito, en este caso basado en J. communis, de Panticosa, Huesca) y en -et -eta en Cataluña o Levante ; concretamente, el microtopónimo La Sabineta, recogido en Albelda (Huesca) y en Valencia, alude a J. phoenicea pero no se trataría necesariamente de un diminutivo, sino de un colectivo equivalente a Savineda.

El sufijo -alis da por todo el ámbito topónimos en -al como Enebral, en Burgo de Osma, etc. y Sabinal, en Cigudosa -ambos en Soria y mayormente basados en J. thurifera- o bien topónimos en -ar como Barranco del Sabinar, en Villanueva de Sigena, Huesca. La terminación -osum da los abundanciales en -osa -oso, castellanos sobre todo, y en -ós catalanoaragoneses. Sirvan de muestra la Val Sabinosa en Pina de Ebro (Zaragoza) y su equivalente 
Valdenebroso (Langa de Duero, Soria), ambos bellos parajes poblados de sabina albar (J. thurifera).

Cabe añadir que hay topónimos «ocultos » etimológicamente, pero cuyo significado etnoecológico nos permite referirlos a sabinas y enebros. A partir del topónimo transparente Dehesa del Sabinar, de Urrea de Gaén, descubrimos la «Dehesa Mayor» de Griegos y Guadalaviar, en Albarracín, o simplemente la Dehesa en Fortanete y otros lugares del Sistema Ibérico, indicando el aprovechamiento ganadero extensivo de esas comunidades de $J$. thurifera. Aparte de los anotados, en la tabla quedan otros por comprobar la especie, por ejemplo la «Dehesa de los Enebrales » cerca de Daroca (Zaragoza) que podría haberse generado por J. communis o J. oxycedrus, pero donde también podría haber $J$. phoenicea. Asimismo, cabría seguir analizando el topónimo Cerna (Calamocha, Teruel), posiblemente generado por la « cierna », es decir, la lluvia lenta del polen de $J$. thurifera, tal como la llaman en Cabrejas (Soria).

Otro tanto ocurre con las decenas de sardas y derivados, más los coscojares o lentiscares, topónimos alusivos a un matorral dominado por Quercus coccifera, Pistacia lentiscus o Rhamnus lycioides en el que no suele faltar la sabina negral, J. phoenicea; este « coscojar aragonés » (Montserrat 1964-1966) se extiende sobre todo por los cerros de la Cuenca del Ebro y lo pastan cabras y ovejas. También los topónimos basados en la voz gayuba, cuya primera acepción nos lleva a la ericácea rastrera Arctostaphylos uva-ursi (L.) Spreng. - la planta entera o su fruto-, podrían relacionarse con las sabinas, pues en los confines de Teruel y Valencia a la recolección de los gálbulos caídos de viejos pies de J. thurifera -para alimentación animal- se le llamaba « recoger gayubas »; en todo caso, la ericácea y la sabina albar coinciden en «gallubares », « gayubosos », etc. de la Cordillera Ibérica, por ejemplo en los municipios turolenses de Alpeñés, El Pobo y Manzanera o en los zaragozanos de Ariza y Atea.

Prueba de la importancia de los Juniperus en el mundo rural ibérico serían los topónimos mayores que han generado, tanto en Aragón como en Castilla. Citemos, a título ilustrativo El Sabinar (Zaragoza), Fuentenebro y Nebreda (Burgos), Valdenebro (Soria), Valdenebro de los Valles (Valladolid), el citado Mas de la Sabina (Valencia), etc. Ahora bien, hay nombres de lugar como Sabiñán (Zaragoza) o Sabiñánigo (Huesca) que pueden llamar a error, ya que su etimología está más relacionada con antropónimos romanos -Sabinius, Sabinianus- que con nuestros árboles o arbustos (Miguel 2011).

Para terminar, mencionemos los hagiotopónimos o advocaciones marianas relacionadas: N. ${ }^{a}$ S. ${ }^{a}$ del Enebral (Moral de Hornuez, Segovia) -con viejos ejemplares de J. thurifera como los que ya llamaron la atención de l'Écluse (1576) en el siglo XVI-, N. ${ }^{a}{ }^{\text {S. }}{ }^{\text {a de los }}$ Enebrales en Tamajón (Guadalajara) o N. ${ }^{a}$ S. ${ }^{a}$ de la Sabina en Farlete (Zaragoza) y Villaespesa (Teruel), lugares separados por una distancia de $200 \mathrm{~km}$ (Morales \& Villar 2003).

\section{A guisa de conclusión}

Gracias a los datos catastrales y a otras diversas fuentes, en Aragón más Castilla y León -regiones que albergan las mayores masas de enebros y sabinas peninsulares- hemos podido recoger 432 topónimos basados en las tres especies del género Juniperus de hoja escuamiforme, y ello sin contar repeticiones, ya que el mismo topónimo puede aparecer en uno o varios municipios. Como era de esperar, las especies de porte arbóreo son las más representadas : J. thurifera -unos 294 topónimos bastante variados- y J. phoenicea, unos 101 topónimos. Finalmente, la especie de porte rastrero J. sabina sólo ha dado 5 topónimos, tres plurales y dos colectivos, todos ellos en las provincias castellanas de Burgos, León y Palencia. Sin duda, la mayor cantidad de topónimos juniperinos de la Península Ibérica se concentra en las provincias de Teruel, Zaragoza y Burgos ; asimismo, el topónimo más extendido es "sabinar», ya que se repite en 27 lugares de Teruel y 7 de Zaragoza, entre otros.

La complejidad producida por las diversas lenguas habladas en el territorio considerado se traduce en los numerosos sinónimos -diferentes nombres para una misma especie- y opuestamente en los casos llamativos de polisemia : un solo nombre puede aludir a una, dos o tres de las especies implicadas, incluso dentro del mismo ámbito lingüístico. Por eso, la interpretación de los diversos fitotopónimos estudiados -mayormente microtopónimos-, resulta difícil y solo se podrá saber la especie 
que los ha generado visitando sobre el terreno el paraje correspondiente.

Las múltiples facetas de los topónimos estudiados, expresadas a través de distintos dominios lingüísticos -occitano, aragonés, castellano, galaico-portugués, etc.-, reflejan que los sabinares y enebrales son formaciones modificadas o favorecidas por el hombre. Tradicionalmente eran bien conocidas, apreciadas y útiles en el seno de los paisajes agro-silvopastorales, aunque ahora se vean más o menos abandonadas en torno a los pueblos y despoblados de las sierras y páramos, por lo general bajo un clima continental de la Península Ibérica.

Llegado el siglo XXI, los nuevos modos de vida hacen que se pierda el significado etnobotánico de muchos de los fitotopónimos mencionados e incluso que se olviden algunos de ellos. Sin embargo, como ya hemos dicho (Sanz \& González 2006; Villar 2010), este patrimonio cultural, bien recopilado e interpretado, contribuirá a comprender mejor la gestión de nuestras áreas forestales en el pasado, su situación actual e incluso su posible evolución en el futuro.

Agradecimientos.- A las Gerencias Territoriales del Catastro en Aragón y Castilla y León, así como al Instituto Geográfico Nacional por habernos cedido amablemente los datos de parajes y nombres geográficos.

\section{Bibliografía}

Aguilella A., 2011. Protegiendo la biodiversidad. Etnobotánica y conservación. Mètode 72 : 53-64.

Alvar M., Llorente A., Buesa T. \& Alvar E., 1980. Atlas lingüístico y etnográfico de Aragón, Navarra y Rioja, t. III : Plantas. Zaragoza, IfC/CsIC, láms. 342484b.

Aymerich P. \& Villar L., 2013. Sur les peuplements de Genévrier thurifère en Haut Pallars (Pyrénées centrales, Catalogne). Présentation géobotanique et écologique. Véase este mismo volumen, p. 41-53.

Barrios J.C., Fuentes M.T. \& Ruiz J.P., 1992. El saber ecológico de los ganaderos de la Sierra de Madrid. Cuadernos madrileños del medio ambiente. Comunidad de Madrid, Madrid, 160 p.

Benito M., 2002. Pueblos del Alto Aragón, el origen de sus nombres. Servicio de Patrimonio Etnológico, Lingüístico y Musical. Diputación General de Aragón. Edición electrónica http://www.aragon.es/edycul/patrimonio/etno/pueblos/indice.htm

BoA, 2011a. Decreto 127/2011, de 31 de mayo, del Gobierno de Aragón, por el que se aprueba el Catálogo de Montes de Utilidad Pública de la Provincia de Huesca. Boletín Oficial de Aragón 115 : 1450214596.
BoA, 2011b. Decreto 128/2011, de 31 de mayo, del Gobierno de Aragón, por el que se aprueba el Catálogo de Montes de Utilidad Pública de la Provincia de Teruel. Boletín Oficial de Aragón 115 : $14597-$ 14657.

Coromines J., 1989-1997. Onomasticon Cataloniae. 8 vols. Ediciones Curial, Barcelona.

Coronado A., 2006. Enebros y sabinas. Un caso de doble inversión semántica. Revista de Soria 53 : 5966.

Costa Tenorio M., García Antón M., Morla Juaristi C. \& Sainz Ollero H., 1990. La evolución de los bosques en la Península Ibérica : una interpretación basada en datos paleobiogeográficos. Ecología, Fuera de Serie : 31-58.

García Sánchez J.J., 2007. Atlas toponímico de España. Arco Libros, Madrid, 407 p.

González Bernáldez F., 1992. Los paisajes del agua. Terminología popular de los humedales. J.M. Reyero Editor, Madrid, 258 p.

Gordaliza F.R. \& Canal J.M., 1993. Toponimia palentina. Caja España, Palencia, 596 p.

L'Écluse Ch. de, 1576. Rariorum aliquot stirpium per hispanias observatarum historia. Amberes. [Edición en español de L.R. Laca \& R. Morales publicada por la Junta de Castilla y León en 2005, bajo el título Descripción de algunas plantas raras encontradas en España y Portugal. IsBN : 84-9718-315-0. Valladolid, 379 p.]

Llorente A., 2003. Toponimia salmantina. Diputación de Salamanca, Salamanca, 311 p.

Miguel P., 2011. Los nombres de Aragón: sus poblaciones. Heraldo de Aragón, Zaragoza, 400 p.

Montserrat P., 1964-1966. Vegetación de la Cuenca del Ebro. P. Cent. Pir. Biol. Exp. 1 (5) : 1-22 + mapa.

Morales R., Macía J.M., Dorda E. \& García Villaraco A., 1996. Nombres vulgares II. Archivos de Flora iberica $7: 1-325$.

Morales R. \& Villar L., 2003. Advocaciones de la Virgen con referencia al mundo vegetal. Revista de Folklore 270 : 212-216.

Pérez-Soba I., 2010. Catálogo de los montes públicos de Zaragoza. Institución Fernando el Católico, Diputación de Zaragoza, 173 p.

Rivas-Martínez S. y coautores, 2011. Mapa de series, geoseries y geopermaseries de vegetación de España. Itinera Geobotanica 18-1 : 1-424 y 18-2 : 425-800.

Ruiz de la Torre J., 2006. Flora Mayor. Organismo Autónomo Parques Nacionales. Ministerio de Medio Ambiente, Madrid, 1756 p.

Sanz Elorza M., 2008. La flora y la fauna en la toponimia segoviana. Estudio sobre el léxico de la naturaleza en la provincia de Segovia. Caja Segovia, Obra Social y Cultural, Segovia, 245 p.

Sanz Elorza M. \& Agón S., 1997. Árboles notables de la provincia de Huesca. Publicaciones y Ediciones del Alto Aragón S.A. Huesca, 251 p.

Sanz Elorza M. \& González Bernardo F., 2008. Toponimia de origen vegetal en la provincia de Segovia y su sentido ecológico y etnobotánico. Lazaroa 27 : 103-125.

Vidaller R., 1989. Dizionario sobre espezies animals y bexetals en o bocabulario altoaragonés. IEA, Huesca, Cosas Nuestras 7 : 1-330. [Reed., 2004. Libro de as matas y os animals. Dizionario aragonés d'espezies animals y bechetals. Consejo de Protección de la Naturaleza de Aragón, Zaragoza, 395 p.] 
Villar L., 2003. Los saberes científico y popular en torno a las plantas del Pirineo Aragonés. Un ejemplo de biodiversidad cultural. Monografías de la Real Academia de Ciencias de Zaragoza $n^{\circ} 23$. Zaragoza, $42 \mathrm{p}$.

Villar L., 2005a. Toponimia de origen vegetal en el Alto Aragón. Los nombres colectivos relacionados con especies arbóreas y su significado ecológico. Alazet $17: 239-264$.

Villar L., 2005b. Toponimia de origen vegetal en el Alto Aragón, II. Sinfitónimos relacionados con arbustos y su sentido ecológico. Flora Montiberica 29 : 4353.

Villar L., 2010. La toponimia vegetal refleja el saber etnoecológico : el caso del Pirineo central (España). In : Pochettino M.L., Ladio A.H. \& Arenas P.M. (eds.), Tradiciones y transformaciones en Etnobotánica. Cyted, San Salvador de Jujuy : 445-456.

Villar L., 2013. Le Genévrier thurifère, vieil arbre compagnon de l'homme en Méditerranée occidentale. Véase este mismo volumen.

Villar L. \& Ferrández J.V., 2000. Usos etnobotánicos de la sabina albar y arbustos que le acompañan en Aragón. In : Gauquelin T., Asmodé J.F. \& Largier G. (eds.), Le Genévrier thurifère (Juniperus thurifera L.) dans le bassin occidental de la Méditerranée : systématique, écologie, dynamique et gestion.
Actes du colloque international de Marignac (Haute-Garonne), 26-27 septembre 1997. Paris, ONF, coll. «Les dossiers forestiers » $6: 130-139$.

Villar L., Palacín J.M., Calvo C., Gómez D. \& Montserrat G., 1987. Plantas medicinales del Pirineo Aragonés y demás tierras oscenses. Diputación Provincial e Instituto Pirenaico de Ecología-Csic, Huesca, 320 p.

Villar L., Sesé J.A. \& Ferrández J.V., 1997. Atlas de la Flora del Pirineo Aragonés, vol. I. Consejo de Protección de la Naturaleza de Aragón e Instituto de Estudios Altoaragoneses, Zaragoza y Huesca, XCI $+648 \mathrm{p}$.

Villar L., Sesé J.A. \& Ferrández J.V., 2001. Atlas de la Flora del Pirineo Aragonés, vol. II. Consejo de Protección de la Naturaleza de Aragón e Instituto de Estudios Altoaragoneses, Zaragoza y Huesca, XXII $+790 \mathrm{p}$.

Yagüe L., Sanz A., Peso C. del, Ordóñez C. \& Pinillos F., 2006. Evolución de las masas puras de Juniperus thurifera L. en Castilla y León a partir de los datos de los inventarios forestales nacionales 2 y 3 (años 1992 y 2002). In : García-González M. D. (coord.), Actas del III coloquio internacional sobre sabinares y enebrales (Género Juniperus). Ecología y Gestión forestal sostenible, Junta de Castilla y León y Comunidad de Madrid, Soria, vol. II : 239-247. 
